\title{
COVID-19 treatments and pathogenesis including anosmia in K18-hACE2 mice
}

https://doi.org/10.1038/s41586-020-2943-z

Received: 5 August 2020

Accepted: 2 November 2020

Published online: 9 November 2020

Check for updates

\author{
Jian Zheng ${ }^{1,4}$, Lok-Yin Roy Wong ${ }^{1,4}$, Kun Li ${ }^{2,4}$, Abhishek Kumar Verma ${ }^{1,4}$, Miguel E. Ortiz ${ }^{2}$, \\ Christine Wohlford-Lenane ${ }^{2}$, Mariah R. Leidinger ${ }^{3}$, C. Michael Knudson ${ }^{3}$, David K. Meyerholz ${ }^{3}$, \\ Paul B. McCray Jr ${ }^{1,2 \bowtie}$ \& Stanley Perlman ${ }^{1,2 \varpi}$
}

\begin{abstract}
The ongoing coronavirus disease 2019 (COVID-19) pandemic is associated with substantial morbidity and mortality. Although much has been learned in the first few months of the pandemic, many features of COVID-19 pathogenesis remain to be determined. For example, anosmia is a common presentation, and many patients with anosmia show no or only minor respiratory symptoms ${ }^{1}$. Studies in animals infected experimentally with severe acute respiratory syndrome coronavirus 2 (SARS-CoV-2), the cause of COVID-19, provide opportunities to study aspects of the disease that are not easily investigated in human patients. Although the severity of COVID-19 ranges from asymptomatic to lethal ${ }^{2}$, most experimental infections provide insights into mild disease ${ }^{3}$. Here, using K18-hACE2 transgenic mice that were originally developed for SARS studies ${ }^{4}$, we show that infection with SARS-CoV-2 causes severe disease in the lung and, in some mice, the brain. Evidence of thrombosis and vasculitis was detected in mice with severe pneumonia. Furthermore, we show that infusion of convalescent plasma from a recovered patient with COVID-19 protected against lethal disease. Mice developed anosmia at early time points after infection. Notably, although pre-treatment with convalescent plasma prevented most signs of clinical disease, it did not prevent anosmia. Thus, K18-hACE2 mice provide a useful model for studying the pathological basis of both mild and lethal COVID-19 and for assessing therapeutic interventions.
\end{abstract}

SARS-CoV-2 uses the same receptor as SARS-CoV, human angiotensin-converting enzyme 2 (hACE2) ${ }^{5}$. Mice, which are susceptible to SARS-CoV, are resistant to infection with SARS-CoV-2 because of incompatibilities between mouse ACE2 and the viral spike protein. During the 2003-2004 SARS epidemic, because infected mice developed only mild disease, we and others engineered mice that transgenically displayed hACE2 $2^{4,6,7}$. K18-hACE2 mice express hACE2 driven by the cytokeratin 18 (KRT18) promoter, predominantly in epithelial cells ${ }^{8}$. K18-hACE2 mice infected with SARS-CoV developed overwhelming encephalitis, and mild virus-induced pneumonia ${ }^{4}$. Both SARS-CoV-2 and SARS-CoV use hACE2 but show differences in disease manifestations in patients. SARS-CoV-2 infects the upper airways to a greater extent than SARS-CoV, and has also been associated with clinical manifestations such as anosmia, ageusia, thrombosis and endothelial damage in the lung vasculature, cardiac and neurological disease, and a multisystem inflammatory disease in children and adolescents ${ }^{1,9,10}$. On the basis of these observations, we reasoned that SARS-CoV-2 infection of the K18-hACE2 mice might also show differences in pathogenesis. Here we demonstrate the use of K18-hACE2 mice for studies of COVID-19 disease and treatments.

\section{Clinical and virological characterization}

To assess the susceptibility of K18-hACE2 mice to SARS-CoV-2, we intranasally challenged male and female mice using inocula of $10^{3}, 10^{4}$ and $10^{5}$ plaque-forming units (PFU). We observed a dose-dependent increase in weight loss and mortality (Fig. 1a). At the $10^{5}$ PFU inoculum, all mice succumbed, whereas all mice survived when challenged with $10^{3} \mathrm{PFU}$. There was variable mortality when mice were inoculated with $10^{4} \mathrm{PFU}$ (2 out of 3 female miceand 3 out of 7 male mice survived). To understand the cause(s) of this morbidity and mortality better, we assessed the tissue distribution of infectious virus and viral genomic RNA at 2,4 and 6 days post infection (dpi) in mice receiving $10^{5} \mathrm{PFU}$ of SARS-CoV-2. The predominant target organs were the lung at early time points and, variably, the brainat later time points. Virus replicated to high titres in lung tissue at 2 dpi and decreased at 4 and 6 dpi (Fig.1b). In some but not all mice, brain titres gradually increased from 2 to $6 \mathrm{dpi}$. Variability in brain infection was also described in a recent study of K18-hACE2 mice infected withSARS-CoV-2 ${ }^{11}$.Small amounts of infectious virus were detected in the kidney, small intestine and colon, perhaps signifyinglow level haematogenous dissemination (Fig.1b). Wealso detected viral RNA in the heart, liver, spleen, kidney, intestine and colon (Extended Data Fig. 1a). These results demonstrate thatSARS-CoV-2 infection of K18-hACE2 mice causes a dose-dependent lethal respiratory illness, with a subset of mice developing brain infection that also contributes to death.

\section{Extensive and progressive lung disease}

In view of the early pulmonary virus replication, we stained lungs at 4 and $6 \mathrm{dpi}$ for SARS-CoV-2 antigen. Using an inoculum of $10^{5} \mathrm{PFU}$, we 

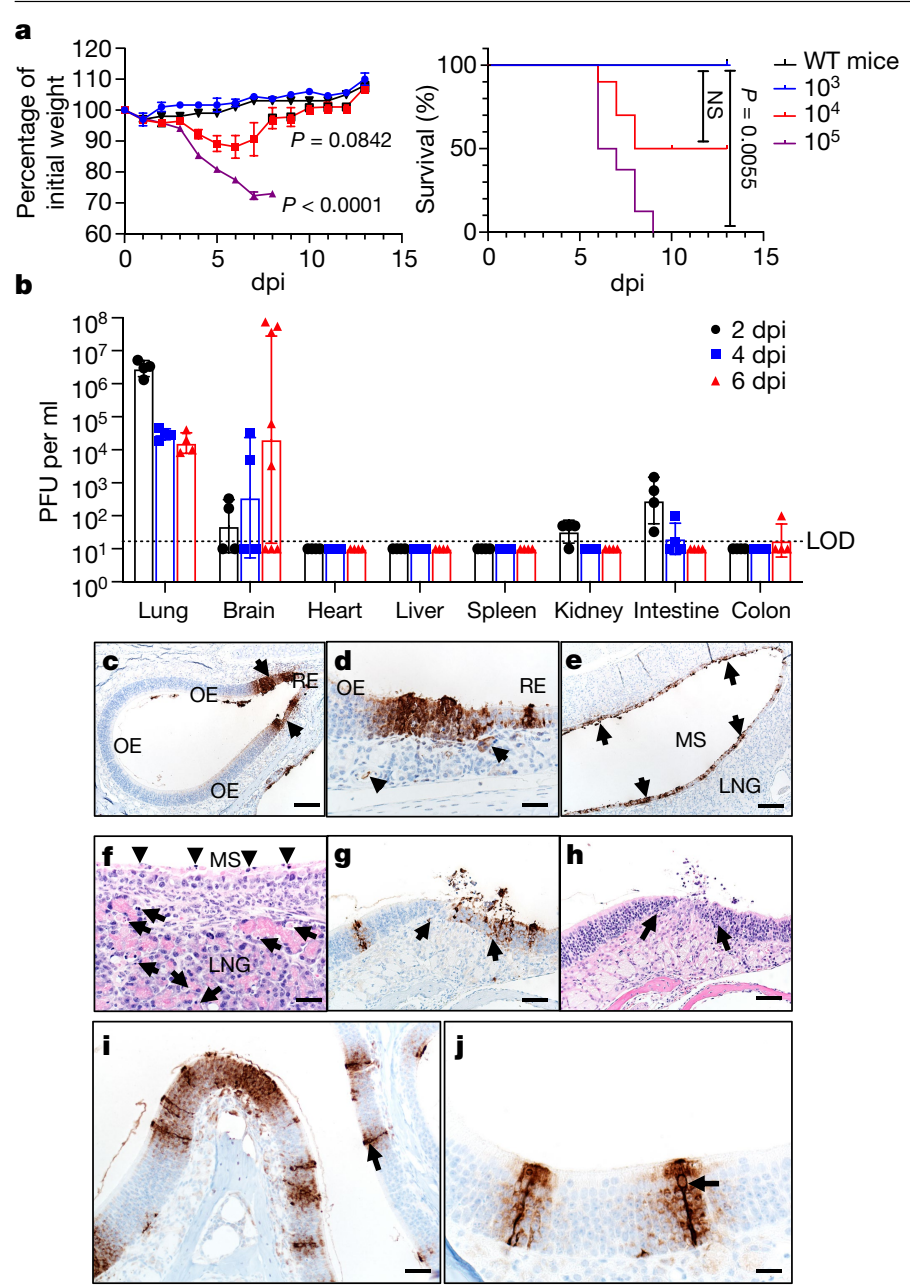

Fig. 1 | Clinical and pathological disease in K18-hACE2 mice infected with SARS-CoV-2. a, Percentage of initial weight and survival of wild-type (WT) $(n=1$ mouse) and K18-hACE2 mice infected with $10^{3}$ ( $n=3$ mice), $10^{4}$ ( $n=10$ mice), or $10^{5}$ ( $n=8$ mice) PFU of SARS-CoV-2 per mouse (two independent experiments). $P$ values determined by analysis of variance (ANOVA), two-tailed Student's $t$-tests without adjustments (weight change) and log-rank (Mantel-Cox) tests (survival). Left, $P<0.000110^{3}$ vs $10^{5} \mathrm{PFU}$ inoculum; $P=0.0842$ for $10^{4} \mathrm{vs} 10^{5} \mathrm{PFU}$ inoculum. NS, not significant. $\mathbf{b}$, Infectious viral titres detected by plaque assay in different organs at 2, 4 and 6 dpi with $10^{5}$ PFU SARS-CoV-2 (days 2, $4 n=4$ mice; day 6, $n=8$ mice (brain) and $n=4$ (other organs); three independent experiments). LOD, limit of detection. $\mathbf{c}-\mathbf{j}$, Nasal and sinus tissues were examined at days $2(\mathbf{c}-\mathbf{f})$ and $5(\mathbf{g}-\mathbf{j})$. Nucleocapsid protein immunostaining $(\mathbf{c}-\mathbf{e}, \mathbf{g}, \mathbf{i}, \mathbf{j})$ and haematoxylin and eosin (H\&E) stain $(\mathbf{f}, \mathbf{h})$. Data in $\mathbf{a}$ and $\mathbf{b}$ are mean \pm s.e.m. c, Nucleocapsid protein immunostaining in olfactory epithelium (OE). d, Nucleocapsid protein immunostaining (brown) localized near the interface of olfactory epithelium and respiratory epithelium (RE). Subjacent to epithelium, nucleocapsid protein was occasionally detected in endothelial lining of vessels (left arrowhead) and Bowman's glands (right arrow) were occasionally detected. e, f, Maxillary sinus (MS) lining epithelium had extensive immunostaining for nucleocapsid protein (e, brown, arrows) with common sloughing and cellular debris (f, arrowheads). The lateral nasal glands (LNG) also had multifocal cellular and karyorrhectic debris (f, arrows). $\mathbf{g}, \mathbf{h}$, Olfactory epithelium at day 5 with nucleocapsid protein immunostaining (g, arrows) localized near interface with respiratory epithelium. At these sites, cellular sloughing and loss of cellularity (h, arrows) were seen. $\mathbf{i}, \mathbf{j}$, Strong immunostaining of nucleocapsid protein (arrows) was seen in tall olfactory epithelium cells with 'classic' morphology of sustentacular cells, along with the immunostain expanding to adjacent cells. Scale bars, $128 \mu \mathrm{m}(\mathbf{c}, \mathbf{e}), 63 \mu \mathrm{m}(\mathrm{g}, \mathbf{h})$, $31 \mu \mathrm{m}(\mathbf{d}, \mathbf{f}), 42 \mu \mathrm{m}(\mathbf{i}), 21 \mu \mathrm{m}(\mathbf{j})$. Two sections of each tissue from 3-4 mice per group were evaluated. observed extensive and diffuse parenchymal localization of SARS-CoV-2 nucleocapsid protein at $4 \mathrm{dpi}$ that was less intense by $6 \mathrm{dpi}$ (Extended Data Fig. 1b). Uninfected tissues showed no nucleocapsid protein signal. Examination of haematoxylin and eosin-stained tissues revealed evidence of diffuse alveolar damage with progressive alveolar or interstitial lesions characterized by oedema, inflammation and focal cytomegaly in some alveolar lining cells (Extended Data Fig. 1c). We also found an accumulation of immune effector cells, including granulocytes and macrophages, evidence of cell death, haemorrhage, hyaline membranes, occasional vascular thrombi (Extended Data Fig. 1c) and rare syncytia. Pulmonary vascular thrombi are often observed in severe cases of human COVID-19 ${ }^{9}$. These findings are summarized in Extended Data Fig. 1d.

We also surveyed liver, heart, spleen, kidney, small intestine and colon tissues for disease-associated changes (Extended Data Fig. 2). At 4 dpi, occasional vessels in the liver had evidence of fibrin thrombi adherent along the vascular wall (Extended Data Fig. 2a), suggestive of the coagulopathy observed in COVID-19 autopsies ${ }^{12}$. The other tissues examined showed no disease-specific changes. To further characterize the lung tissue responses to SARS-CoV-2 infection, we assessed the expression of several cytokines, chemokines and innate immune transcripts at 2, 4 and 6 dpi by quantitative PCR with reverse transcription (qRT-PCR). As shown in Extended Data Fig. 3a, there were significant increases in type I, II and III interferons, ISG-15, RIG-I, MDA5, IL-1ß, IL-6, IL-8, IL-12, CCL-2, CCL-5, CCR7, CXCL-2, CXCL-9, CXCL-10 and TNF compared with uninfected lungs over the course of infection. Similar changes in the expression of pro-inflammatory molecules were observed in patients with COVID-19 ${ }^{13}$.

\section{Inflammatory cell infiltration}

To further investigate the factors that contribute to lung injury and virus clearance, we performed immunophenotyping of infiltrating cells at 4 and 6 dpi (Extended Data Fig. 3b-e). We observed a progressive increase in the numbers of macrophages, monocytes, neutrophils and CD4 and CD8 T cells (Extended Data Fig. 3e). Infiltrating inflammatory macrophages, monocytes and neutrophils may have both protective and pathogenic roles, on the basis of previous studies of SARS and Middle East respiratory syndrome (MERS) ${ }^{14}$. Virus-specific CD8 and CD4 T cells, which are required for optimal clearance of SARS-CoV-2, were detected in lung tissues at $6 \mathrm{dpi}$. These cells responded to pools of peptides representing the SARS-CoV-2 spike, nucleocapsid and membrane proteins to varying extents, as assessed by the expression of IFN $\gamma$ and TNF (Extended Data Fig. 3c, d). Together, these results indicate that lung disease in K18-hACE2 mice shares features with severe COVID19. Another characteristic of severe human COVID-19 is an early antibody response, consistent with plasmablast activation ${ }^{13}$. We detected serum-neutralizing antibodies as early as $6 \mathrm{dpi}$, consistent with such a response (Extended Data Fig. 3f).

\section{Brain infection in some mice}

Because infectious virus was detected in the brains of 5 out of 8 mice after $10^{5}$ PFU intranasal inoculation, we immunostained tissues for viral nucleocapsid protein at 4 and $6 \mathrm{dpi}$. We observed no antigen staining at $4 \mathrm{dpi}$ ( 4 out of 4 brains studied), but saw extensive staining at $6 \mathrm{dpi}$ in several brain regions including the olfactory bulb, cerebral cortex, caudate/putamen, thalamus, hypothalamus and ventral striatum ( 2 out of 3 brains studied) (Extended Data Fig. $4 a$, Extended Data Table 1). Uninfected mice showed no nucleocapsid protein staining ( 3 out of 3 mice). Haematoxylin and eosin staining showed cell death and thrombi, noted in the thalamus (Extended Data Fig. 4b, c) and foci of cell death were sometimes also detected adjacent to degenerative ependyma cells (Extended Data Fig. 4d). The thalamus is affected in many patients with COVID-19 with 

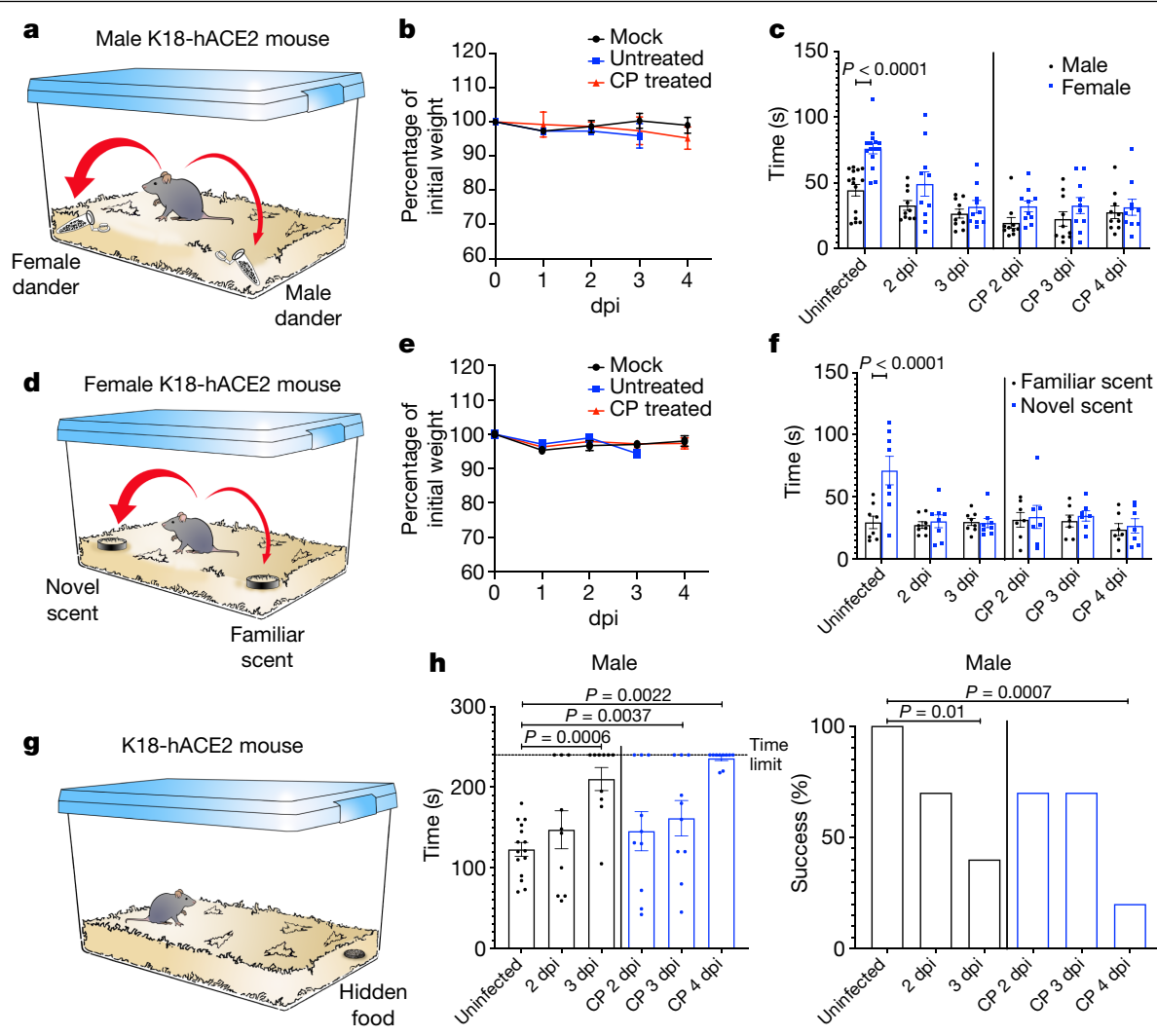$$
\text { h }
$$
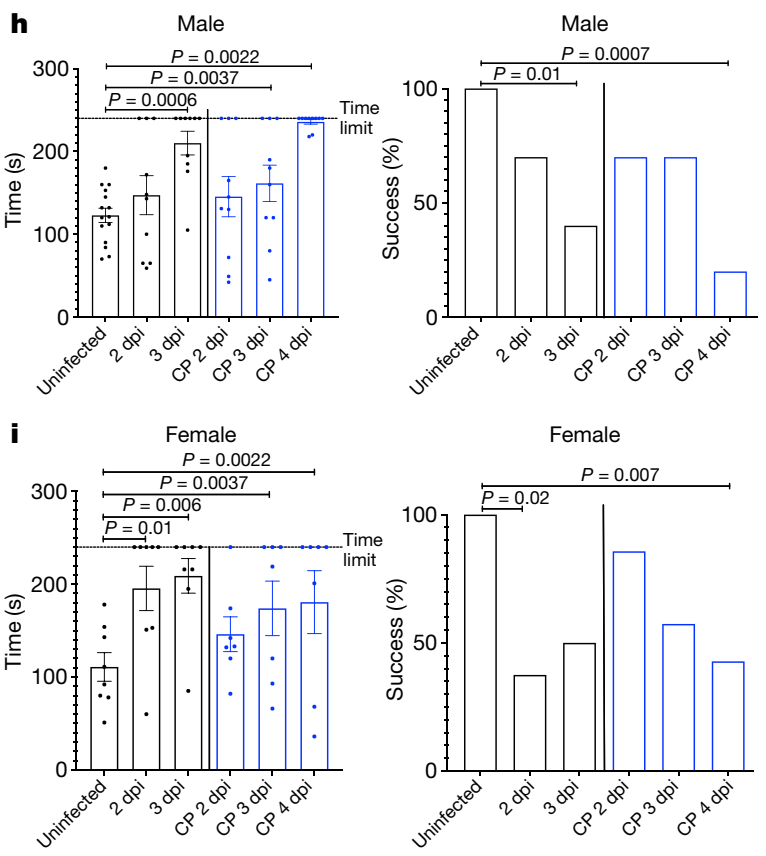

Fig. 2 |SARS-CoV-2 infection causes anosmia in K18-hACE2 mice. Male and female mice were treated with PBS (uninfected) or $10^{5}$ PFU of SARS-CoV-2 intranasally. a, d, Schematic showing social scent-discrimination tests.b,e, Weights were recorded daily.c, Each male mouse was allowed $5 \mathrm{~min}$ in the cage with scents from male or female mice (bedding from male or female cages) placed at two corners of the cage. The time that male mice spent sniffing male or female scents was recorded. $P$ values were determined by two-way ANOVA. f, Female mice were exposed to their own bedding (familiar) or bedding from another cage (novel). The time that female mice spent exploring each bedding was recorded. $P$ values were determined by two-way ANOVA. In some experiments, mice were pre-treated with undiluted convalescent plasma (CP; results on right of vertical line) (c, f). $\mathbf{g}-\mathbf{i}$, Buried food test (schematic

neurological disease ${ }^{15}$. Of note, although most of the affected areas are secondary or tertiary connections of the olfactory bulb, some-such as the area postrema and hypoglossal nucleus-are not directly connected. Similar results were observed in analyses of mice infected with SARS-CoV ${ }^{16}$. These results suggest an important role for olfactory bulb infection in spread to the brain in some mice but also indicate that virus may enter the central nervous system by other routes. Infection of the olfactory epithelium is expected to precede brain infection and could also contribute to the anosmia and ageusia observed in many patients ${ }^{1}$, including some who were asymptomatic or mildly symptomatic. shown in g). Food was buried under the bedding and each mouse was allowed $4 \mathrm{~min}$ in the cage to search for the food. $\mathbf{h}$, The dotted line denotes the time limit of 4 min. $P$ values were determined by two-tailed Mann-Whitney $U$-test. $\mathbf{i}$, The percentage of mice that found the buried food within 4 min is shown. $P$ values were determined by $\chi^{2}$ Fisher's exact test. In some experiments, mice were pre-treated with undiluted convalescent plasma (denoted by blue bars in $\mathbf{h}, \mathbf{i}$ ). Male mice: uninfected: $n=15$ mice; infected: $n=10$ mice. Female mice: uninfected and infected: $n=8$ mice each; infected $/$ plasma-treated: $n=7$ mice; analysed in four independent experiments. Data are mean \pm s.e.m. Preference indices for the social scent-discrimination assays are shown in Extended Data Fig. 5.

\section{Infection of the sinonasal epithelium}

To assess virus replication in the upper respiratory tract after intranasal inoculation, we quantified viral RNA in nasal secretions by qRT-PCR at 3 dpi. SARS-CoV-2 genomic $\operatorname{RNA}\left(C_{\mathrm{t}}=21.5 \pm 1.2\right.$, mean \pm s.e.m. $)$ was detected in secretions from 5 out of 7 mice, and 4 of these mice had evidence of subgenomic RNA $(36.7 \pm 1.1)$, consistent with active virus replication. Viral antigen was readily detected in both the respiratory and olfactory epithelium at 2 and $5 \mathrm{dpi}$. At $2 \mathrm{dpi}$, virus antigen was present at several sites, often at the interface of the olfactory and respiratory epithelium (Fig. 1c). Viral antigen was also detected in nerve bundles 

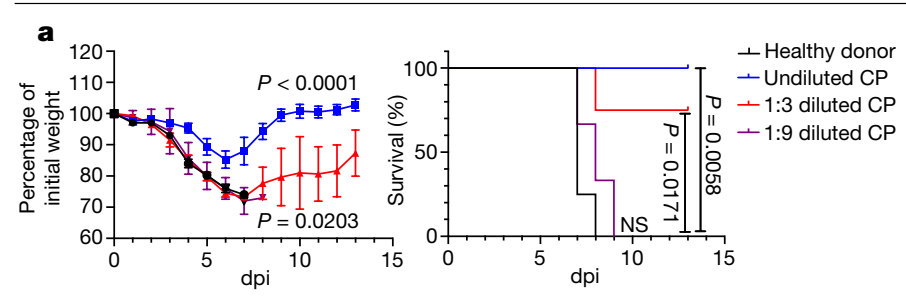

b Lung
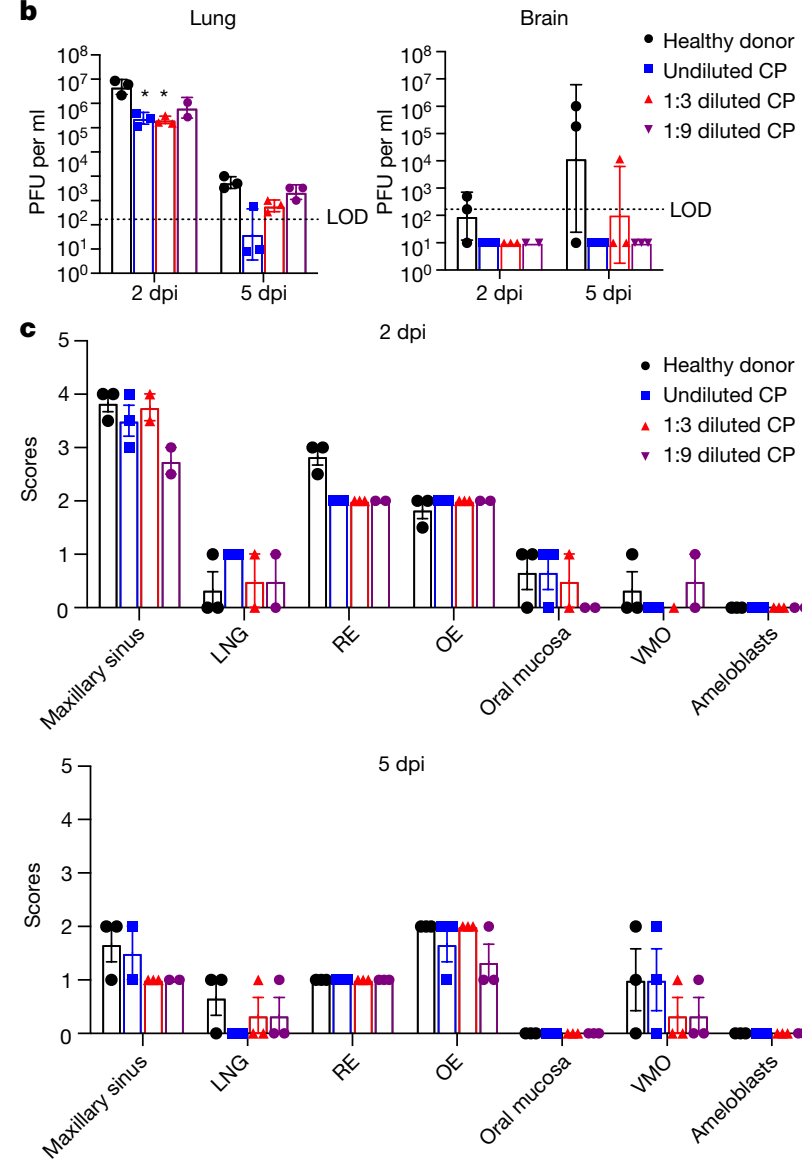

2 dpi

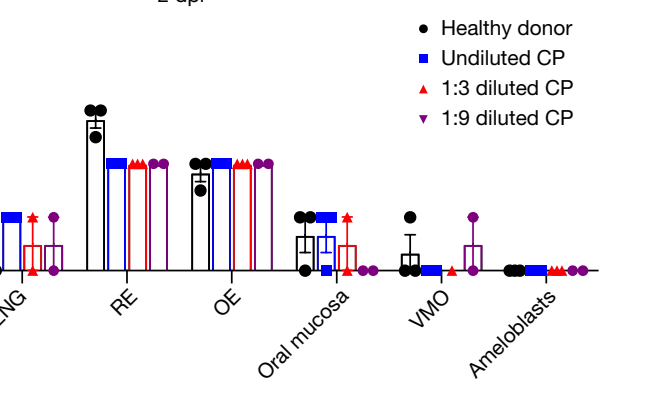

$5 \mathrm{dpi}$

Fig. 3 | Effects of convalescent plasma on outcomes. a, Percentage of initial weight (left) and survival (right) of K18-hACE2 mice receiving control serum ( $n=4$ mice, black), undiluted ( $n=4$ mice, blue), 1:3 dilution ( $n=4$ mice, red) and $1: 9$ dilution ( $n=3$ mice, purple) human convalescent plasma $(C P)$ at 24 h before challenge with $10^{5} \mathrm{PFU}$ of SARS-CoV-2. Data are from two independent experiments. $P$ values were determined by ANOVA, two-tailed Student's $t$-tests without adjustments (weight change), and log-rank (Mantel-Cox) tests (survival).b, Viral titres of plasma-treated mice in the lungs (left) and brains (right) at 2 and 5 dpi. $n=3$ mice, except $n=2$ mice at 2 dpi, $1: 9$ dilution; 1 independent experiment. LOD, limit of detection. ${ }^{*} P=0.0455$, control vs undiluted $\mathrm{CP} ;{ }^{*} P=0.0443$ control vs $1: 3$ diluted $\mathrm{CP}$, two-tailed Student's $t$-tests without adjustments. c, Scores of nucleocapsid protein immunostaining in plasma-treated mice in the nasal cavity at $2 \mathrm{dpi}$ (top) and $5 \mathrm{dpi}$ (bottom). 0 , none; 1 , rare $<1 \% ; 2$, multifocal or localized $<33 \%$ cells; 3 , multifocal, coalescing, $33-66 \% ; 4$, extensive $>67 \%$. Two sections of each sinonasal cavity from three mice per group were evaluated. Data are mean \pm s.e.m. VMO, vomeronasal organ.

subjacent to the olfactory epithelium (Extended Data Fig. 4e) and occasionally in vascular endothelia (Fig. 1d, left arrow) and Bowman's glands (Fig. 1d, right arrow). At sites of antigen positivity in the olfactory epithelium and maxillary sinus, we observed cell death and cellular debris at 2 dpi (Fig. 1e, f, Extended Data Fig. 4f), which progressed to cell sloughing and loss of cellularity by $5 \mathrm{dpi}$ (Fig. 1g, h). ACE2 has been detected in sustentacular cells in the olfactory epithelium ${ }^{17}$, but not in olfactory sensory neurons, suggesting that these cells are a primary site of infection. Consistent with this, we detected SARS-CoV-2 antigen in sustentacular cells (Fig. 1i, j). Infection of sustentacular cells is not expected to result in spread to the olfactory bulb and its connections, but could still contribute to anosmia.

\section{Anosmia in infected K18-hACE2 mice}

To directly assess anosmia, we performed three sets of behavioural tests, all of which require a normal sense of smell, as described in the Methods. First, in the social scent-discrimination assays, male mice were exposed to bedding containing female or male dander in a 2-ml Eppendorf tube (Fig. 2a). Mice identify the tube visually, and then preferentially spend time with the female dander, if olfaction is normal. Because female mice are not preferentially attracted to male dander ${ }^{18}$, in a second experiment, female mice were exposed to bedding from their home cage ('familiar') and another, foreign cage ('novel') (Fig. 2d). Mice with normal olfaction preferentially explore the unfamiliar bedding. Third, we used a buried food test, in which mice are attracted to a food item that is hidden in the bedding that they were previously conditioned to detect (Fig. 2g). K18-hACE2 mice infected with SARS-CoV-2 performed worse than control mice in all of the tests so that at days 2 and 3 after infection, male mice spent less time in the vicinity of the female dander (Fig. 2c), female mice were not attracted to the foreign bedding (Fig. $2 \mathrm{f}$ ) and male and female mice took longer to find buried food (Fig. 2h, i). At 2 and 3 dpi, brains were not infected (Fig. 1b, Extended Data Fig. 4a) and mice exhibited minimal weight loss (Fig. 2b, e). In addition, mobility was largely normal as there were no significant differences in the amount of time spent in exploring the tube containing male dander (Fig. 2c) or familiar bedding (Fig. 2f), when infected or uninfected mice were compared. Preference indices were calculated for the social scent-discrimination assays and confirmed hyposmia or anosmia (Extended Data Fig. 5). Together, these results suggest that hyposmia or anosmia at these times points is primarily caused by infection of the nasal epithelium and is not a consequence of generalized malaise or the spread of virus to the brain.

\section{Human convalescent plasma therapy}

Convalescent plasma from survivors of COVID-19 is being tested as a treatment in patients with clinical COVID-19 ${ }^{19}$. Studies have shown positive effects of convalescent plasma on outcomes in several infectious diseases, if titres are sufficiently high and if the convalescent plasma is administered early in the course of disease $\mathrm{e}^{20}$. To assess the effect of convalescent plasma on outcomes in K18-hACE2 mice infected with SARS-CoV-2, we administered undiluted and diluted human high-titre plasma (original neutralizing titre 1:1,480) intravenously $12 \mathrm{~h}$ before infection. Undiluted plasma administered $12 \mathrm{~h}$ before infection protected mice from death but not mild weight loss and reduced lung tissue titres, whereas convalescent plasma diluted 1:3 provided partial protection (Fig. 3a, b). Pre-treatment with undiluted and diluted convalescent plasma markedly inhibited the spread of infection to the brain (Fig. 3b) even in mice that died, supporting the conclusion that a lethal lung infection was the cause of death. On examination of lung tissues, levels of viral antigen and pathological changes were greatly decreased by convalescent plasma treatment (Extended Data Fig. 6a). Delivery of undiluted plasma at $\mathbf{2 4}$ hours after infection was partially protective (Extended Data Fig. 6b). Notably, convalescent plasma did not diminish infection of sinonasal tissues (Fig. 3c). Finally, to assess the effects of convalescent plasma treatment on SARS-CoV-2-induced anosmia, we treated infected mice with plasma at $12 \mathrm{~h}$ before infection and assessed mice for olfactory loss as described above (Fig. 2). Even though mice had minimal signs of clinical disease after convalescent plasma treatment, by day 4 all mice exhibited profound anosmia (Fig. 2c, f, h, i, Extended Data Fig. 5). 


\section{Discussion}

K18-hACE2 mice infected with SARS-CoV-2 developed dose-dependent lung disease with features that are similar to severe human COVID-19, including diffuse alveolar damage, inflammatory cell infiltration, tissue injury, lung vascular damage and death. hACE2 transgenic mice driven by other promoters have also been developed, with variable outcomes observed after SARS-CoV-2 infection ${ }^{21,22}$. Notably, K18-hACE2 mice also support SARS-CoV-2 replication in the sinonasal epithelium and develop anosmia, a common feature of human disease. Furthermore, the uniformly fatal disease outcome with a $10^{5}$ inoculum was prevented by pre-treatment with convalescent plasma from a patient with COVID-19. Notably, pre-treatment with plasma did not prevent initial infection of the lungs, damage to nasal respiratory and olfactory epithelia, or anosmia. We observed greater anosmia in female mice than in male mice in the presence or absence of convalescent plasma. Similarly, anosmia is more common in women than in men with COVID-19 $9^{23-25}$.SARS-CoV-2 infection of K18-hACE2 mice treated with convalescent plasma or, potentially, neutralizing monoclonal antibodies will be especially useful for studies of anosmia because mice do not succumb to the infection but, similar to many infected patients with mild disease, have olfactory loss as a major manifestation ${ }^{1}$. Anosmia appears to result from initial infection and damage to supporting sustentacular cells rather than to olfactory sensory neurons, which suggests that the resulting inflammatory milieu, rather than direct neuronal damage, is disease-causing. Therefore, we postulate that the observed anosmia has at least two possible, non-exclusionary explanations. First, the infection of sustentacular cells may disrupt signalling from olfactory sensory neurons to the olfactory bulb. For example, sustentacular cells provide support to olfactory sensory neurons by maintaining ion balance ${ }^{26}$, which may be altered either directly by infection to sustentacular cells or indirectly via disorganization of the olfactory epithelium, leading to loss of cilia from olfactory sensory neurons and deficient signal transduction ${ }^{27,28}$. The second possibility is collateral damage to olfactory sensory neurons resulting from the secretion of pro-inflammatory cytokines by sustentacular cells infected with SARS-CoV-2. These cells have been reported to secrete cytokines and chemokines such as TNF, IL-1 $\beta$, IL- $1 \alpha$ and CXCL2 in patients with chronic rhinosinusitis and COVID-1929,30. Together, K18-hACE2 mice infected with SARS-CoV-2, readily available from Jackson Laboratories, recapitulate many of the findings observed in patients with COVID-19 and provide a useful model to study pathogenesis and to evaluate interventions.

\section{Online content}

Any methods, additional references, Nature Research reporting summaries, source data, extended data, supplementary information, acknowledgements, peer review information; details of author contributions and competing interests; and statements of data and code availability are available at https://doi.org/10.1038/s41586-020-2943-z.

1. Spinato, G. et al. Alterations in smell or taste in mildly symptomatic outpatients with SARS-CoV-2 infection. J. Am. Med. Assoc. 323, 2089-2090 (2020).
2. Williamson, E. J. et al. Factors associated with COVID-19-related death using OpenSAFELY Nature 584, 430-436 (2020).

3. Muñoz-Fontela, C. et al. Animal models for COVID-19. Nature 586, 509-515 (2020).

4. McCray, P. B., Jr et al. Lethal infection in K18-hACE2 mice infected with SARS-CoV. J. Virol. 81, 813-821 (2006).

5. Zhou, P. et al. A pneumonia outbreak associated with a new coronavirus of probable bat origin. Nature 579, 270-273 (2020)

6. Tseng, C. T. et al. SARS coronavirus infection of mice transgenic for the human angiotensin-converting enzyme 2 (hACE2) virus receptor. J. Virol. 81, 1162-1173 (2006).

7. Yang, X. H. et al. Mice transgenic for human angiotensin-converting enzyme 2 provide a model for SARS coronavirus infection. Comp. Med. 57, 450-459 (2007).

8. Chow, Y. H. et al. Development of an epithelium-specific expression cassette with human DNA regulatory elements for transgene expression in lung airways. Proc. Natl Acad. Sci. USA 94, 14695-14700 (1997).

9. Ackermann, M. et al. Pulmonary vascular endothelialitis, thrombosis, and angiogenesis in Covid-19. N. Engl. J. Med. 383, 120-128 (2020).

10. Feldstein, L. R. et al. Multisystem inflammatory syndrome in U.S. children and adolescents. N. Engl. J. Med. 383, 334-346 (2020).

11. Winkler, E. S. et al. SARS-CoV-2 infection of human ACE2-transgenic mice causes severe lung inflammation and impaired function. Nat. Immunol. 21, 1327-1335 (2020).

12. Bradley, B. T. et al. Histopathology and ultrastructural findings of fatal COVID-19 infections in Washington State: a case series Lancet 396, 320-332 (2020).

13. Mathew, D. et al. Deep immune profiling of COVID-19 patients reveals distinct immunotypes with therapeutic implications. Science 369, eabc8511 (2020).

14. Channappanavar, R. et al. Dysregulated type I interferon and inflammatory monocyte-macrophage responses cause lethal pneumonia in SARS-CoV-infected mice. Cell Host Microbe 19, 181-193 (2016).

15. Poyiadji, N. et al. COVID-19-associated acute hemorrhagic necrotizing encephalopathy: imaging features. Radiology 296, E119-E120 (2020).

16. Netland, J., Meyerholz, D. K., Moore, S., Cassell, M. \& Perlman, S. Severe acute respiratory syndrome coronavirus infection causes neuronal death in the absence of encephalitis in mice transgenic for human ACE2. J. Virol. 82, 7264-7275 (2008).

17. Bilinska, K., Jakubowska, P., Von Bartheld, C. S. \& Butowt, R. Expression of the SARS-CoV-2 entry proteins, ACE2 and TMPRSS2, in cells of the olfactory epithelium: identification of cell types and trends with age. ACS Chem. Neurosci. 11, 1555-1562 (2020).

18. Dey, S. et al. Cyclic regulation of sensory perception by a female hormone alters behavior. Cell 161, 1334-1344 (2015).

19. Joyner, M. et al. Early safety indicators of COVID-19 convalescent plasma in 5,000 patients. J. Clin. Invest. https://doi.org/10.1172/JCl140200 (2020)

20. Casadevall, A., Joyner, M. J. \& Pirofski, L. A. SARS-CoV-2 viral load and antibody responses: the case for convalescent plasma therapy. J. Clin. Invest. 130, 5112-5114 (2020).

21. Bao, L. et al. The pathogenicity of SARS-CoV-2 in hACE2 transgenic mice. Nature $\mathbf{5 8 3}$, 830-833 (2020).

22. Jiang, R. D. et al. Pathogenesis of SARS-CoV-2 in transgenic mice expressing human angiotensin-converting enzyme 2. Cell 182, 50-58 (2020).

23. Makaronidis, J. et al. Seroprevalence of SARS-CoV-2 antibodies in people with an acute loss in their sense of smell and/or taste in a community-based population in London, UK an observational cohort study. PLoS Med. 17, e1003358 (2020).

24. Giacomelli, A. et al. Self-reported olfactory and taste disorders in patients with severe acute respiratory coronavirus 2 infection: a cross-sectional study. Clin. Infect. Dis. 71, 889-890 (2020).

25. Lechien, J. R. et al. Olfactory and gustatory dysfunctions as a clinical presentation of mild-to-moderate forms of the coronavirus disease (COVID-19): a multicenter European study. Eur. Arch. Otorhinolaryngol. 277, 2251-2261 (2020).

26. Vogalis, F., Hegg, C. C. \& Lucero, M. T. Ionic conductances in sustentacular cells of the mouse olfactory epithelium. J. Physiol. (Lond.) 562, 785-799 (2005).

27. Bryche, B. et al. Massive transient damage of the olfactory epithelium associated with infection of sustentacular cells by SARS-CoV-2 in golden Syrian hamsters. Brain Behav. Immun. 89, 579-586 (2020).

28. Zhang, A. J. et al. SARS-CoV-2 infects and damages the mature and immature olfactory sensory neurons of hamsters. Clin. Infect. Dis. https://doi.org/10.1093/cid/ciaa995 (2020)

29. Imamura, F. \& Hasegawa-Ishii, S. Environmental toxicants-induced immune responses in the olfactory mucosa. Front. Immunol. 7, 475 (2016).

30. Torabi, A. et al. Proinflammatory cytokines in the olfactory mucosa result in COVID-19 induced anosmia. ACS Chem. Neurosci. 11, 1909-1913 (2020).

(c) The Author(s), under exclusive licence to Springer Nature Limited 2020 


\section{Methods}

No statistical methods were used to predetermine sample size. The experiments were not randomized, and investigators were not blinded to allocation during experiments and outcome assessment unless stated otherwise.

\section{Human subject approval}

Written informed consent was obtained from subjects to obtain plasma for participation in this study. The study was approved by the Institutional Review Board of the University of Iowa (IRB (202003554 and 201402735).

\section{Mice, cells and virus}

Transgenic mice expressing hACE2 were previously reported ${ }^{4}$. hACE2 is expressed under control of the cytokeratin 18 (KRT18) promoter. Although the $K R T 18$ promoter predominantly directs gene expression to multiple epithelia-lined tissues ${ }^{8}$, it also directs reporter gene expression in neurons ${ }^{31}$. The mice (7-8 weeks old, male or female) used in these studies were obtained from the Jackson Laboratory (034860-B6. $\mathrm{Cg}$-Tg(K18-ACE2)2Prlman/J) and are congenic on the C57BL/6 background. Non-transgenic $\mathrm{C} 57 \mathrm{BL} / 6$ mice were used as controls in some experiments. All protocols were approved by the Institutional Animal Care and Use Committee of the University of Iowa. The 2019n-CoV/ USA-WA1/2019 strain of SARS-CoV-2 (accession number: MT985325.1) used in these studies was passaged on Calu-32B4 cells (ATCC HTB-55). Calu-32B4 cells were grown in MEM (GIBCO) supplemented with 20\% FBS. Vero E6 cells (ATCC CRL-1586) were grown in DMEM (GIBCO) supplemented with $10 \%$ fetal bovine serum (FBS).

\section{Infection of mice}

Mice were lightly anaesthetized with ketamine/xylazine and infected intranasally with the indicated amount of SARS-CoV-2 in a total volume of $50 \mu$ l DMEM. Mouse weight and health were monitored daily. All experiments with SARS-CoV-2 were performed in a Biosafety Level 3 (BSL3) Laboratory at the University of lowa.

Nasal lavage. Mice were sedated with ketamine/xylazine $\left(100 \mathrm{mg} \mathrm{kg}^{-1}\right.$ ketamine $/ 12.5 \mathrm{mg} \mathrm{kg}^{-1}$ xylazine). One-hundred microliters of carbachol $\left(100 \mu \mathrm{g} \mathrm{ml}^{-1}\right)$ was administered intraperitoneally and after approximately $15 \mathrm{~min}$, nasal secretions were recovered. Secretions of $20 \mu \mathrm{l}$ were recovered per mouse. Nasal secretions were immediately added to 500 $\mu \mathrm{l}$ Trizol, mixed and stored at $-80^{\circ} \mathrm{C}$ until RNA was isolated.

\section{Viral titre by plaque assay}

Virus or tissue homogenate supernatants were serially diluted in DMEM. 12-well plates of VeroE6 cells were inoculated at $37^{\circ} \mathrm{C}$ in $5 \% \mathrm{CO}_{2}$ for $1 \mathrm{~h}$ and gently rocked every $15 \mathrm{~min}$. After removing the inocula, plates were overlaid with $0.6 \%$ agarose containing $2 \%$ FBS. After 3 days, overlays were removed, and plaques were visualized by staining with $0.1 \%$ crystal violet. Viral titres were quantified as PFU per ml tissue.

\section{Flow cytometry}

The following monoclonal antibodies were used as described by manufacturer at a dilution of 1:200: anti-mouse CD3e-BV421 (clone 145-2C11, 562600), anti-mouse CD16/32 (clone 93, cat. no.:101302), anti-mouse CD4-PercP (clone RM4-5, 550954), anti-mouse CD8-APC-Cy7 (clone 53-6.7, 100714), anti-mouse CD220-APC (clone RA3-6B2, 553092), anti-mouse Ly6C-PerCP (clone HK1.4,128028), anti-mouse Ly6G-FITC (clone 1A8, 127606), anti-mouse CD11b-BV510 (clone M1/70,101263), anti-mouse CD11c-BV421 (clone N418,117343), anti-mouse CD64-PE-Cy7 (X54-5/7.1, 139314), Biolegend; anti-mouse CD103-APC (clone 2E7, 17-1031-80), anti-mouse TNF-FITC (clone MP6-XT22, 11-7321-82), anti-mouse IFN $\gamma$-APC (clone XMG1.2, 25-7311-82), eBioscience. For intracellular cytokine staining (ICS), lymphocytes were cultured in 96-well dishes at $37^{\circ} \mathrm{C}$ for 5-6 $\mathrm{h}$ in the presence of $2 \mu \mathrm{M}$ peptide pool and brefeldin A (BD Biosciences). Cells were then labelled for cell-surface markers, fixed/permeabilized with Cytofix/Cytoperm Solution (BD Biosciences), and labelled with anti-IFN $\gamma$ and anti-TNF antibody. All flow cytometry data were acquired using a BD FACSVerse and analysed with FlowJo software.

\section{Histology and immunohistochemistry}

Mice were anaesthetized and perfused transcardially with PBS, followed by zinc formalin. Lungs were fixed in zinc formalin. For routine histology, tissue sections (approximately $4 \mu \mathrm{m}$ each) were stained with H\&E. The following criteria were used for scoring oedema, hyaline membrane formation and necrotic cellular debris: 0 , none; 1 , uncommon detection in $<5 \%$ lung fields $(200 \times) ; 2$, detectable in up to $33 \%$ of lung fields; 3 , detectable in up to $33-66 \%$ of lung fields; 4 , detectable in $>66 \%$ of lung fields. For scoring neutrophil infiltration: 0 , within normal limits; 1 , scattered PMNs sequestered in septa; 2 , no. 1 plus solitary PMNs extravasated in airspaces; 3 , no. 2 plus small aggregates in vessel and airspaces. For scoring mononuclear infiltrates, thrombosis and haemorrhage: 0 , none; 1 , uncommon detection in $<5 \%$ lung fields $(200 \times) ; 2$, detectable in up to $33 \%$ of lung fields; 3 , detectable in up to $33-66 \%$ of lung fields; 4 , detectable in $>66 \%$ of lung fields.

For SARS-CoV-2 antigen detection, slides were incubated with blocking reagent $(10 \%$ normal goat serum $\times 30 \mathrm{~min})$ followed by rabbit monoclonal antibody against SARS-CoV-2 nucleocapsid protein (1:20,000 dilution $\times 60 \mathrm{~min}, 40143-\mathrm{R} 019$, Sino Biological), then incubated with Rabbit Envision (Dako) and diaminobenzidine (Dako) as chromogen. Tissues were examined and scored in a post-examination method of masking by a boarded experimental pathologist ${ }^{32}$. Ordinal scores for lesion parameters were assigned using the following tiers: 0 , within expected limits; 1 , uncommon, $<5 \% ; 2$, detectable in 5-33\%; 3 , detectable in $34-66 \%$ and 4 , detectable in $>66 \%$ of lung fields $(200 \times$ objective magnification).

\section{Human convalescent plasma}

High-titre convalescent plasma was collected with subject consent under an IRB (202003554)-approved protocol that allows use of samples for research. The convalescent plasma donor was a 58-year-old female patient who had molecularly confirmed COVID-19 more than 4 weeks before their donation. After the donation, she tested positive for HLA antibodies so the plasma was not eligible for administration to patients and was diverted to research. Antibody testing (EUROIMMUN SARS-COV-2 ELISA (IgG)) performed on this donor was 9.8, well above the cut-off value of 1.1 for a positive result. Neutralization titre using a luciferase-expressing SARS-CoV-2 spike protein pseudovirus assay showed that the neutralization half-maximal inhibitory concentration $\left(\mathrm{IC}_{50}\right)$ titre was 1:1,480. Control plasma was obtained from an expired plasma unit collected before COVID-19 spread in our area and this product was collected under an IRB (201402735) approved protocol that allows for research use of these products. Convalescent and control plasma were administered intravenously at the indicated times.

\section{RNA isolation and $q R T-P C R$}

Total RNA was extracted from tissues (or nasal lavage) using Trizol (Invitrogen) according to the manufacturer's protocol. After a DNase treatment step 200 ng of total RNA was used as a template for first strand cDNA. The resulting cDNA was subjected to amplification of selected genes by real-time quantitative PCR using Power SYBR Green PCR Master Mix (Applied Biosystems). Average values from duplicates of each gene were used to calculate the relative abundance of transcripts normalized to HPRT and presented as $2^{-\Delta C t}$. The primers used for cytokines and chemokines were previously reported ${ }^{33}$. For detection of viral genomes, the following primers were used to amplify the genomic RNA for the nucleocapsid protein: 2019-nCoV_N1-F: 5'-GACCCCAAAATCAGCGAAAT-3'; 
2019-nCoV_N1-R: 5'-TCTGGTTACTGCCAGTTGAATCTG-3'. The following primers were used to amplify the subgenomic RNA for the E protein: F: 5'-CGATCTCTTGTAGATCTGTTCTC-3'; R: 5'-ATATTGCAGCAGTACGCACACA-3'.

\section{Pseudovirus neutralizing antibody assay}

To determine neutralization activity of patient and mouse plasma, we used a luciferase reporter-based pseudovirus neutralization assay, which has a nonreplicative vesicular stomatitis virus backbone coated with the SARS-CoV-2 spike protein. Sera from K18-hACE2 mice infected with SARS-CoV-2 were collected at the indicated time points and heat inactivated by incubation at $56^{\circ} \mathrm{C}$ for $30 \mathrm{~min}$. Human convalescent plasma or mouse sera were serially diluted twofold in 96-well plates and incubated with the same volume of VSV pseudotyped with SARS-CoV-2 spike protein at $37^{\circ} \mathrm{C}$ for $1 \mathrm{~h}$. Samples were placed onto Vero E6 cells and incubated at $37^{\circ} \mathrm{C}$ for $1 \mathrm{~h}$ to allow virus binding. After removal of the initial inoculum, cells were incubated for $24 \mathrm{~h}$. Neutralization was measured with a luciferase assay kit (Promega) and plotted relative to the value for control wells.

\section{Social scent-discrimination tests}

For male mice, the social scent-discrimination task was designed to assess the capability of mice to discriminate social scents as previously described $^{34}$. Two identical tubes were separately sealed in a Ziploc bag in a large cage (used for housing rats $(31 \mathrm{~cm} \times 26 \mathrm{~cm} \times 22 \mathrm{~cm})$ ) containing bedding from female mice and from the home (male) cage overnight. Next, mice infected with SARS-CoV-2 or treated with PBS were released in a fresh cage containing the tubes placed at two different corners. Sniffing latency (sniffing and exploring the tubes) was calculated within a 5-min period. One trial on each mouse was performed each day with the position of tubes changed daily. Data were recorded in the BSL3 facility from a distance of approximately $1 \mathrm{~m}$.

For female mice, two identical 3-cm dishes were separately sealed with mouse dander from the home cage ('familiar') and another cage ('novel') overnight. The dander-containing dishes were placed at two corners of a fresh cage. Mice infected with SARS-CoV-2 or treated with PBS mice were released into the cage and sniffing latency was calculated and recorded as described for male mice. One trial on each mouse was performed each day with the position of the dishes changed daily.

We calculated a preference index for each mouse, to compensate for any differences due to decreased mobility or malaise. The preference index for male mice is calculated as: (female time-male time)/female time + male time), whereas for female mice the preference index is: (novel scent time - familiar scent time)/(novel scent time + familiar scent time). Data were analysed using Mann-Whitney $U$-tests.

\section{Buried food test}

Evaluation of odour detection was carried out by a buried food test as previously described ${ }^{34}$. In brief, food palatability was confirmed before initiation of the experiments. SARS-CoV-2-infected or PBS-treated mice were sensitized to the food for $10-15$ min on the day of the experiment. The food pellet was then buried approximately $1 \mathrm{~cm}$ below the surface of bedding in a fresh cage. One trial per infected or uninfected mouse per day was performed with the position of the food changed on a daily basis. The latency to locate and uncover the buried food pellet was recorded with a stopwatch. Mice were allowed to explore the cage for $4 \mathrm{~min}$ and if they could not locate the food, the time was recorded as $4 \mathrm{~min}$. The experiment was repeated daily.

\section{Statistical analysis}

Differences in mean values between groups were analysed by ANOVA and Student's $t$-tests and differences in survival were analysed by log-rank (Mantel-Cox) tests using GraphPad Prism 8. All results are expressed as mean \pm s.e.m. and were corrected for multiple comparisons. For behavioural studies, data from the social scent-discrimination tests, and buried food test were analysed by two-way ANOVA and nonparametric (Mann-Whitney $U$-tests) tests, respectively. $P<0.05$ were considered statistically significant. ${ }^{*} P \leq 0.05,{ }^{* *} P \leq 0.005,{ }^{* * *} P \leq 0.0005$, ${ }^{* * * *} P \leq 0.0001$.

\section{Reporting summary}

Further information on research design is available in the Nature Research Reporting Summary linked to this paper.

\section{Data availability}

The data supporting the findings of this study are documented within the paper and are available from the corresponding authors upon request. Source data are provided with this paper.

31. Chow, Y. H. et al. Targeting transgene expression to airway epithelia and submucosal glands, prominent sites of human CFTR expression. Mol. Ther. 2, 359-367 (2000).

32. Meyerholz, D. K. \& Beck, A. P. Principles and approaches for reproducible scoring of tissue stains in research. Lab. Invest. 98, 844-855 (2018).

33. Li, K. et al. Middle East respiratory syndrome coronavirus causes multiple organ damage and lethal disease in mice transgenic for human dipeptidyl peptidase 4. J. Infect. Dis. 213, 712-722 (2016).

34. Yang, M. \& Crawley, J. N. Simple behavioral assessment of mouse olfaction. Curr. Protoc. Neurosci. Chapter 8, Unit 8.24 (2009)

Acknowledgements We thank M. Moye-Rowley for help with the artwork. This work is supported in part by grants from the US National Institutes of Health (NIH) (P01 AIO60699 (S.P., P.B.M.) and RO1 Al129269 (S.P.)); and the Pathology Core, which is partially supported by the Center for Gene Therapy for Cystic Fibrosis (NIH grant P3O DK-54759), and the Cystic Fibrosis Foundation. P.B.M. is supported by the Roy J. Carver Charitable Trust.

Author contributions Conceptualization and writing of the original draft: P.B.M., S.P. Data curation: A.K.V. Formal analysis: J.Z., L.-Y.R.W., K.L., M.E.O., A.K.V., D.K.M., P.B.M. and S.P. Investigation: J.Z., L.-Y.R.W., K.L., M.E.O., C.W.-L., A.K.V., C.M.K., D.K.M., P.B.M. and S.P. Visualization: A.K.V., M.R.L., D.K.M. Resources: D.K.M., P.B.M. and S.P. Writing, reviewing and editing: J.Z., L.-Y.R.W., S.P. and P.B.M.

Competing interests The authors declare no competing interests.

\section{Additional information}

Supplementary information is available for this paper at https://doi.org/10.1038/s41586-0202943-z.

Correspondence and requests for materials should be addressed to P.B.M. or S.P. Peer review information Nature thanks Dan Barouch, Linda J. Saif, Gonzalo Otazu, and the other, anonymous, reviewer(s) for their contribution to the peer review of this work. Peer reviewer reports are available.

Reprints and permissions information is available at http://www.nature.com/reprints. 

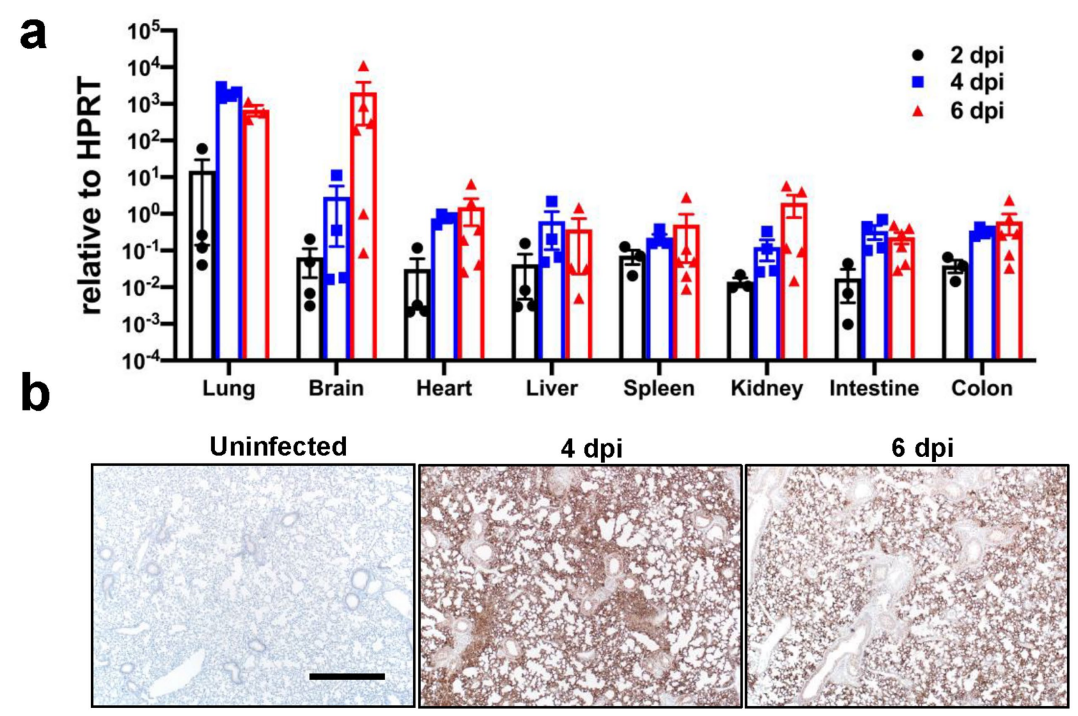

C

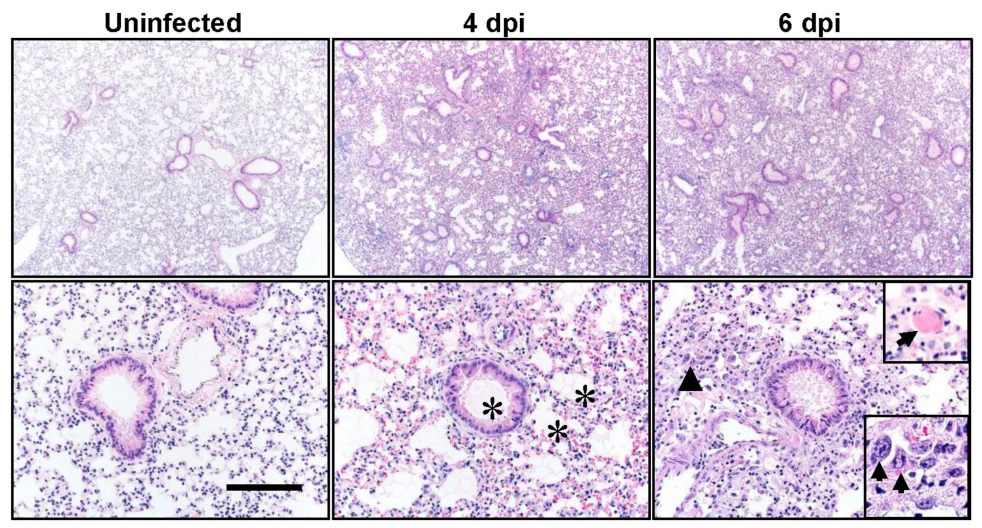

d

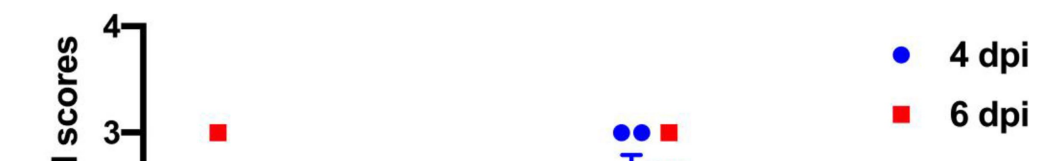

Extended Data Fig. 1 | See next page for caption. 
Extended Data Fig. 1 | Clinical and pathological disease in K18-hACE2 mice infected with SARs-CoV-2. Mice were infected intranasally with $10^{5} \mathrm{PFU}$ of SARS-CoV-2. a, Viral RNA detected by qPCR targeting viral nucleocapsid gene with normalization to $\mathrm{Hprt}$ for the indicated organs at 2,4 and $6 \mathrm{dpi}\left(10^{3}, n=4\right.$; $10^{4}, n=4 ; 10^{5}, n=6$, each organ was collected from an individual mouse). $\mathbf{b}$, Lungs from uninfected $(n=3)$, and infected ( $4 \mathrm{dpi}(n=4)$ and $6 \mathrm{dpi}(n=3))$ mice were immunostained to detect SARS-CoV-2 nucleocapsid protein. Scale bar, $701 \mu \mathrm{m}$. c, Infected lungs exhibited evidence of airway oedema (asterisks, bottom middle panel), alveolar hyaline membranes, vascular thrombosis (top inset and arrow, bottom right panel), dying cells with pyknotic to karyorrhectic nuclei, and proliferative alveolar epithelium (arrowhead and lower inset, bottom right panel, arrows). Scale bar, 701 and $70 \mu \mathrm{m}$ (top and bottom, respectively), H\&E stain. $d$, Summary of lung lesion scoring, as described in Methods (uninfected, $n=3$; day 4, $n=4$; day 6, $n=3$, each brain was collected from an individual mouse). Two sections of each lung from 3-4 mice per group were evaluated. Data in $\mathbf{a}, \mathbf{d}$ are representative of three independent experiments. Data are mean \pm s.e.m. 


\section{Article}

a

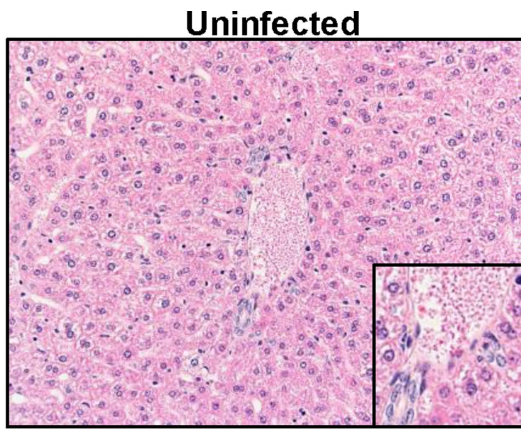

$4 \mathrm{dpi}$

$6 \mathrm{dpi}$

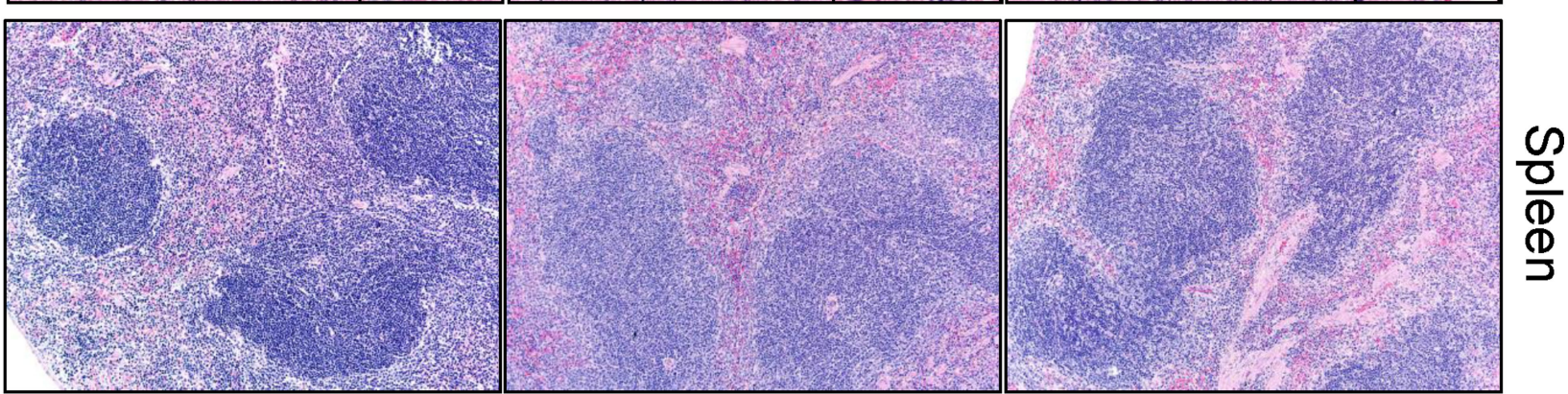

C

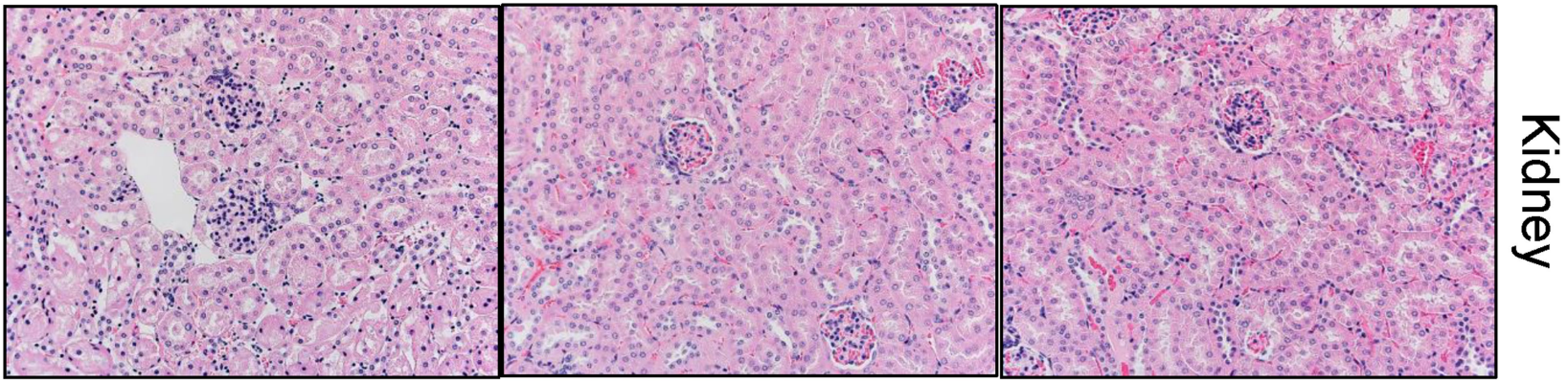

d
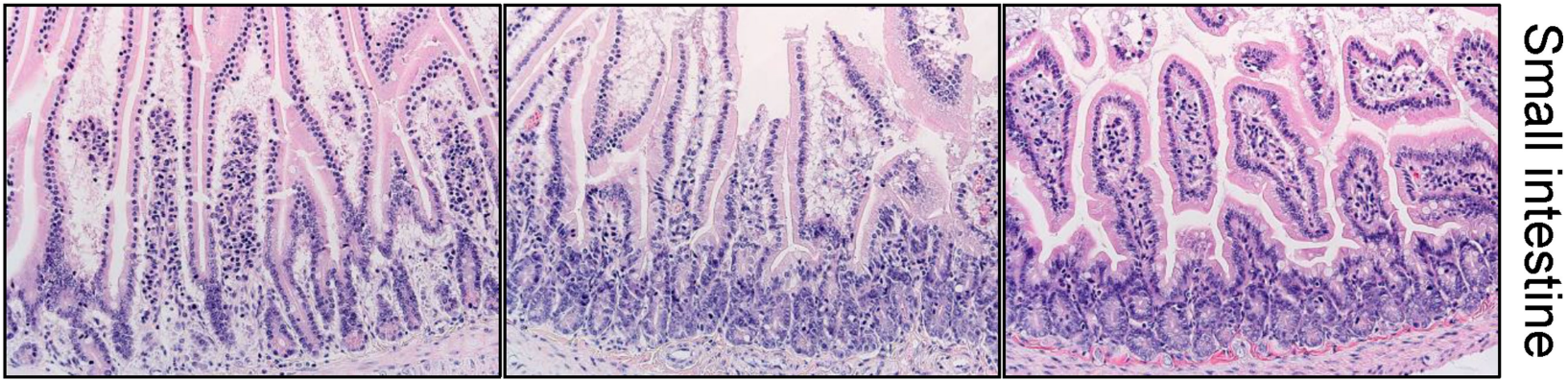

e

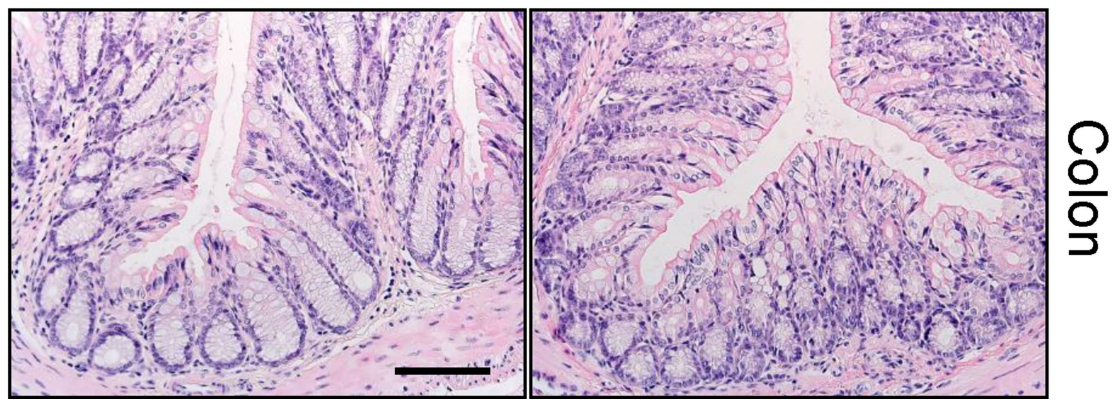

Extended Data Fig. 2 | Histological analysis of extrapulmonary tissue. Mice were killed at 0,4 and $6 \mathrm{dpi}$ with $10^{5} \mathrm{PFU}$ of SARS-CoV-2 and tissues were prepared for histological examination. a-e, Liver (a), spleen (b), kidney (c), small intestine (d) and colon (e) were studied. Pathological changes were minor and only observed in the liver. In the liver, all mice had some blood vessels filled with clear space or aggregates variably composed of erythrocytes or platelets (insets). Rare vessels had evidence of eosinophilic fibrillar material adherent along the vascular wall consistent with fibrin thrombi (a, arrows, inset, middle). Scale bars, $110 \mu \mathrm{m}(\mathbf{a}, \mathbf{c}-\mathbf{e})$ and $221 \mu \mathrm{m}(\mathbf{b}), \mathrm{H} \& \mathrm{E}$ stain. Two sections of each organ from 3-4 mice per group were evaluated. 

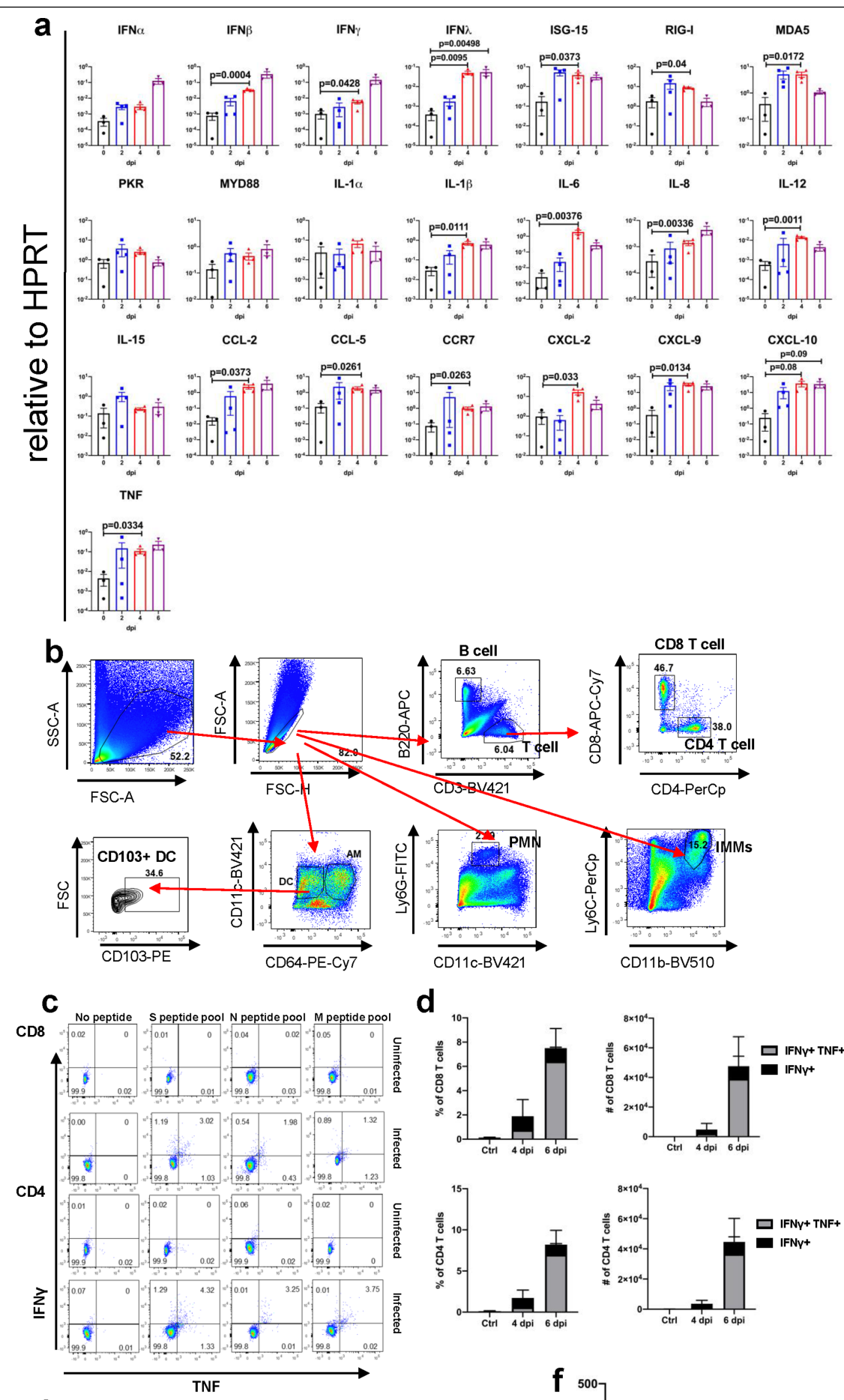

d
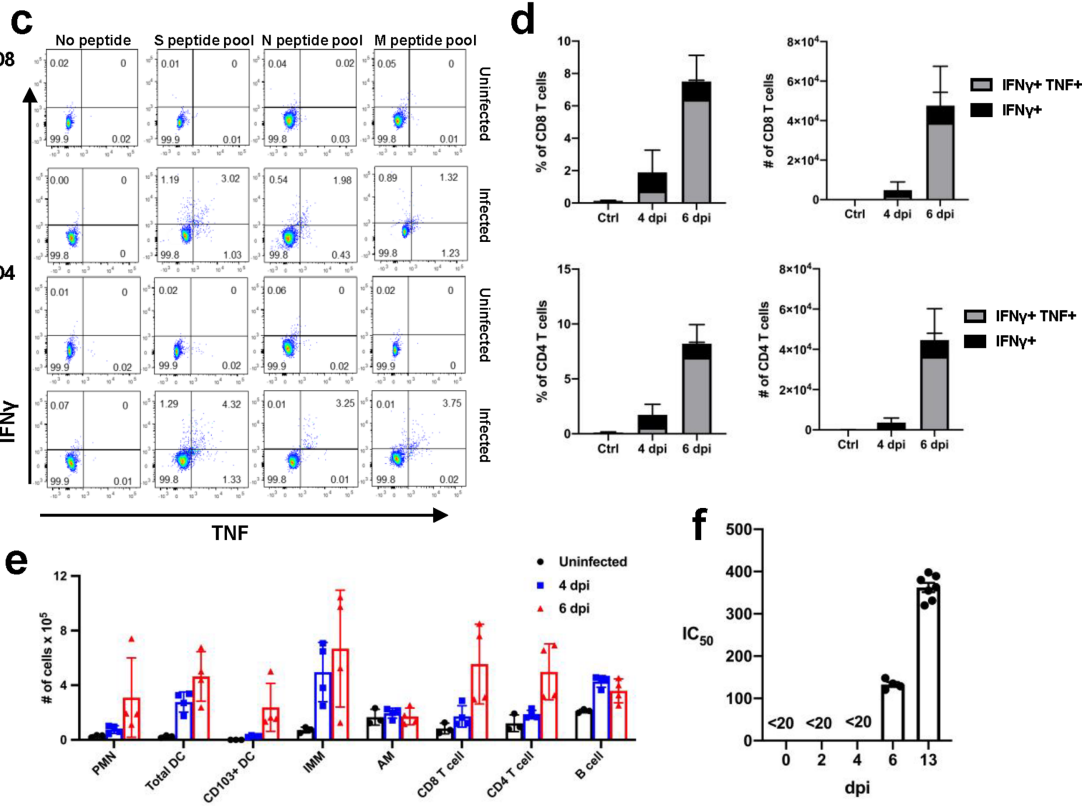

Extended Data Fig. 3 |See next page for caption. 


\section{Article}

Extended Data Fig. 3 | Inflammatory mediators and immune effector cells in infected lungs. a, Cytokine and chemokine transcripts were measured by qRT-PCR after isolation of RNA from the lungs of K18-hACE2 mice infected with $10^{5}$ PFU of SARS-CoV-2 (mock and $6 \mathrm{dpi}, n=3 ; 2$ and $4 \mathrm{dpi}, n=4$, each lung was collected from an individual mouse); one independent experiment. Statistical significance compared to results obtained at $0 \mathrm{dpi}$. $P$ values were determined by two-tailed Student's $t$-tests without adjustments. b, Gating strategy for identification of immune cells in lungs is shown.c, Representative FACS plot of IFN $\gamma^{+} \mathrm{TNF}^{+} \mathrm{CD} 8$ and CD4 $\mathrm{T}$ cells (as gated in $\mathbf{b}$ ) after stimulation with indicated peptide pools in the lungs of K18-hACE2 mice infected with $10^{5}$ PFU of SARSCoV-2.d, Summary data are shown ( $n=3$ mice per time point). Data are representative of two independent experiments. e, Quantification of immune cells (as gated in b) in the lungs ( $n=3$ for uninfected group; $n=4$ for 4 and $6 \mathrm{dpi}$, each lung was collected from an individual mouse). Data are representative of two independent experiments. f, Sera were collected from infected mice at the indicated time points and $\mathrm{IC}_{50}$ values were determined by neutralization of SARS-CoV-2 pseudoviruses expressing luciferase ( $n=4$, days $0,2,4,6 ; n=7$, day 13,2 independent experiments). Data are mean \pm s.e.m. 

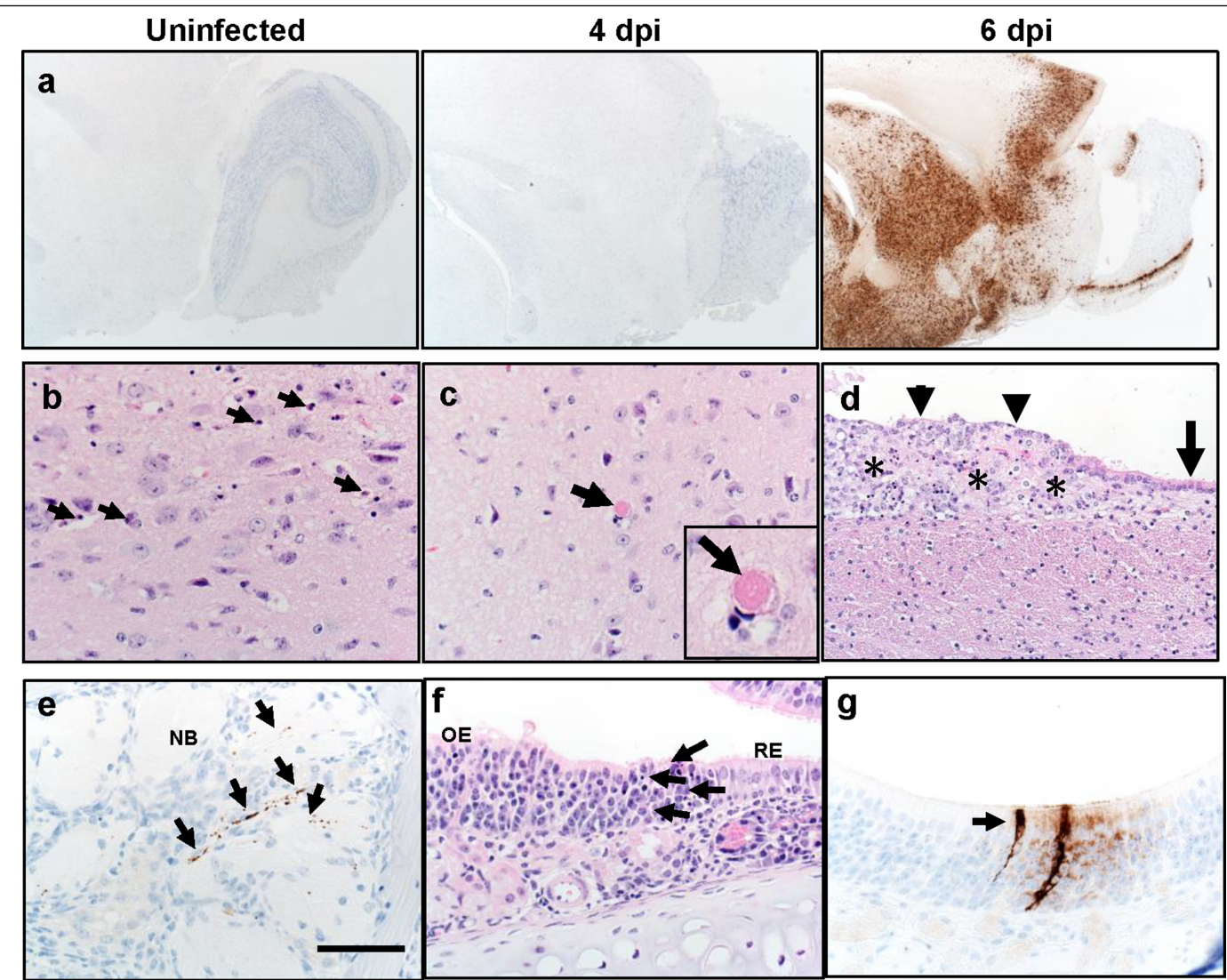

Extended Data Fig. 4 | Brain and nasal cavity infection in SARS-CoV-2 infected K18-hACE2 mice. a, Brains from uninfected and infected $\left(10^{5} \mathrm{PFU}\right.$ of SARS-CoV-2) mice were immunostained to detect SARS-CoV-2 nucleocapsid protein. b, c, Several sites were characterized by cellular and karyorrhectic nuclear debris (b, arrows). Thrombi were detected, seen here in thalamus (c, arrow and inset). Scale bar, $17 \mu \mathrm{m}$. d, Ependyma (arrow) at $6 \mathrm{dpi} \mathrm{had} \mathrm{focal}$ denudation and degeneration of ependymal cells (arrowheads) overlying focal region of cellular and karyorrhectic nuclei debris (asterisks). e, Nerve bundles
(NB) subjacent to olfactory epithelium had evidence of punctate to linear immunostaining (brown, arrows).f, Sites of nucleocapsid protein localization at interface of olfactory epithelium and respiratory epithelium at $2 \mathrm{dpi}$ (see Fig.1d) had evidence of cell death and cellular debris (arrows). g, Olfactory epithelium with sustentacular cell immunostaining for nucleocapsid protein. Scale bar, $1.3 \mathrm{~mm}(\mathbf{a}), 65 \mu \mathrm{m}(\mathbf{b}, \mathbf{c}, \mathbf{e}-\mathbf{g})$ and $130 \mu \mathrm{m}(\mathbf{d})$. Two sections of each tissue from 3-4 mice per group were evaluated. 
a

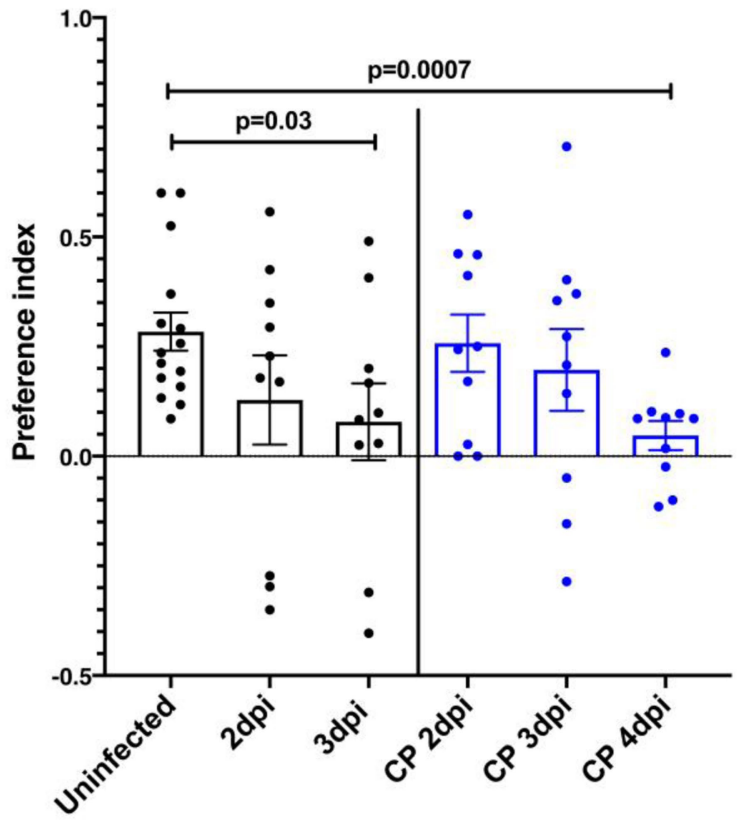

Extended Data Fig. $5 \mid$ Preference indices for social scent-discrimination assays. Preferences indices were calculated as described in Methods for each mouse shown in Fig. 2. The preference index was calculated as time spent with preferred or non-preferred scent: (preferred - non-preferred)/(preferred + non preferred). a, For male mice, preferred scent $=$ female dander; b Female

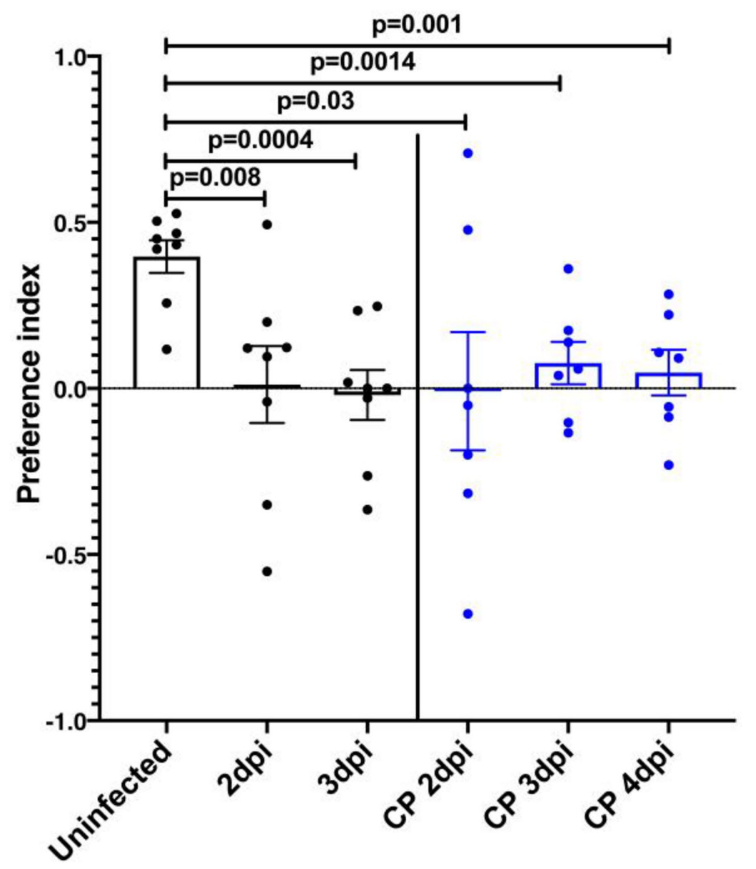

non-preferred $=$ male dander (uninfected: $n=15$ mice, other groups: $n=10$ mice).b, For female mice, preferred scent $=$ novel scent;

non-preferred $=$ familiar scent ( uninfected and infected alone groups: $n=8$ mice each; infected/plasma-treated groups: $n=7$ mice). $P$ values were determined by two-tailed Mann-Whitney $U$-tests. Data are mean \pm s.e.m. 


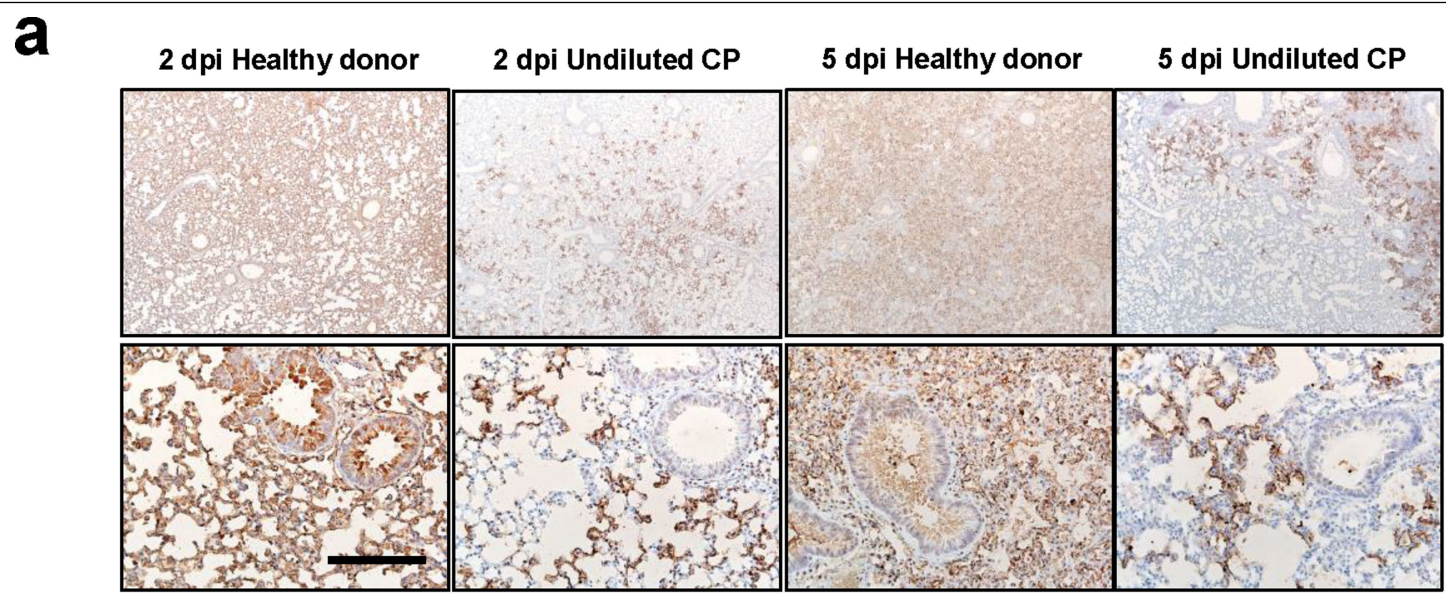

b

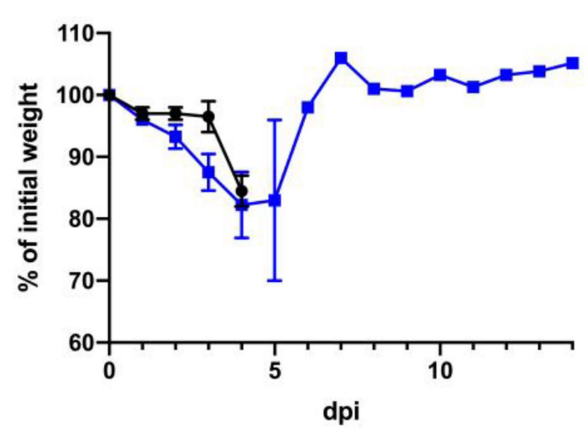

Extended Data Fig. 6 | Effects of convalescent plasma delivered $6 \mathrm{~h}$ before or $24 \mathrm{~h}$ after infection. a, Immunostaining of nucleocapsid protein in the lungs of mice treated with control plasma (healthy donor) or convalescent plasma at 2 and $5 \mathrm{dpi}$ with $10^{5}$ PFU SARS-CoV-2. Scale bars, $800 \mu \mathrm{m}$ (top) and $160 \mu \mathrm{m}$ (bottom). Two sections of each lung from three mice per group were evaluated. Representative images are shown. b, Percentage of initial

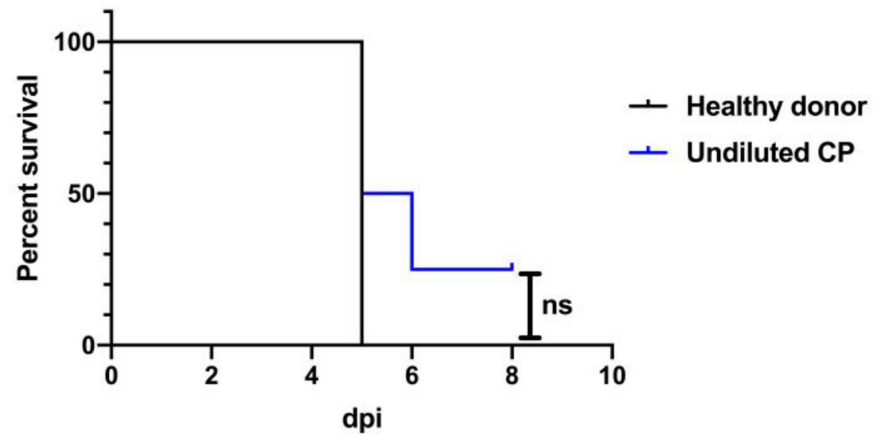

weight (left), and survival (right) of K18-hACE2 mice receiving control plasma $(n=2)$ or undiluted convalescent plasma $(n=4)$ at $24 \mathrm{~h}$ after challenge with $10^{5}$ PFU of SARS-CoV-2. Data are mean \pm s.e.m. and are representative of one independent experiment. $P$ values were determined by ANOVA, two-tailed Student's $t$-tests without adjustments (weights) and log-rank (Mantel-Cox) tests (survival). 
Extended Data Table 1 | Distribution of SARS-CoV2 antigen in brain

\begin{tabular}{|c|c|c|c|}
\hline Olfactory Bulb & & Thalamus & \\
\hline Main Olfactory Bulb & & Ventral Posterolateral Nucleus & +++ \\
\hline Glomerular Layer & - & Ventral Posteromedial Nucleus & +++ \\
\hline Granule Layer & - & Ventral Medial Nucleus & ++ \\
\hline Inner Plexiform Layer & - & Ventral Anterior-Lateral Complex & +++ \\
\hline Mitral Layer & + & Anterior group of the Dorsal Thalamus & +++ \\
\hline Outer Plexiform Layer & - & Medial group of the Dorsal Thalamus & +++ \\
\hline & & Midline group of the Dorsal Thalamus & +++ \\
\hline Accessory Olfactory Bulb & & Reticular Nucleus & +++ \\
\hline Glomerular Layer & - & Geniculate Group & +++ \\
\hline Granular Layer & - & & \\
\hline Mitral Layer & ++ & $\begin{array}{l}\text { Hypothalamus } \\
\text { Supraoptic Nucleus }\end{array}$ & +++ \\
\hline AON Dorsal & +++ & Paraventricular Nucleus & +++ \\
\hline AON Medial & ++ & Arcuate Nucleus & +++ \\
\hline AON lateral & ++ & Suprachiasmatic Nucleus & +++ \\
\hline Taenia Tecta & + & Subfornical Organ & +++ \\
\hline & & Anterior Nucleus & +++ \\
\hline & & Premammillary Nucleus & +++ \\
\hline Midbrain & & Hind Brain & \\
\hline Superior Colliculus & ++ & Pons & \\
\hline Inferior Colliculus & ++ & Nucleus of Lateral Lemniscus & ++ \\
\hline Nucleus Brachium & ++ & Trigeminal Nucleus & ++ \\
\hline Nucleus Sagulum & ++ & Parabrachial Nucleus & ++ \\
\hline Para Bigeminal Nucleus & ++ & Dorsal Tegmental Nucleus & ++ \\
\hline Midbrain Trigeminal Nucleus & ++ & Pontine Nuclei & ++ \\
\hline Substantia Nigra & +++ & Supratrigeminal Nucleus & ++ \\
\hline Ventral Tegmental Area & ++ & Superior Central Nucleus Raphe & ++ \\
\hline Reticular Nucleus & +++ & Locus Ceruleus & ++ \\
\hline Cuneiform Nucleus & ++ & Laterodorsal Tegmental Nucleus & ++ \\
\hline Red Nucleus & ++ & & \\
\hline Oculomotor Nucleus III & ++ & Medulla & \\
\hline Edinger-Westphal Nucleus & ++ & Area Postrema & \\
\hline Trochlear Nucleus IV & ++ & Cochlear Nuclei & ++ \\
\hline Ventral Tegmental Nucleus & ++ & Dorsal Column Nuclei & ++ \\
\hline Pedunculopontine Nucleus & ++ & External Cuneate Nucleus & + \\
\hline & & Nucleus Ambiguus & ++ \\
\hline & & Solitary Tract Nucleus & ++ \\
\hline & & Lateral Reticular Nucleus & ++ \\
\hline & & Magnocellular Reticular Nucleus & ++ \\
\hline & & Medullary Reticular Nucleus & ++ \\
\hline & & Parvicellular Reticular Nucleus & ++ \\
\hline & & Nucleus $\mathrm{X}$ & ++ \\
\hline & & Hypoglossal Nucleus XII & ++ \\
\hline Hippocampus & $(+)$ & & \\
\hline Cerebellum & - & & \\
\hline
\end{tabular}

Four brains were analysed at 6 dpi. '+<+++<+++' denotes relative density of antigen presence. '-' denotes no antigen detected. ' + ' represents presence of antigen in some samples only. AON,

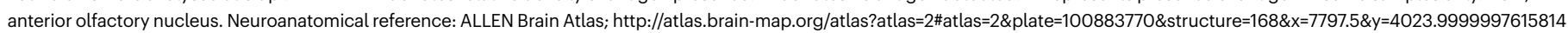
\&zoom=-3\&resolution=10.47\&z=5. 


\section{Reporting Summary}

Nature Research wishes to improve the reproducibility of the work that we publish. This form provides structure for consistency and transparency in reporting. For further information on Nature Research policies, see Authors \& Referees and the Editorial Policy Checklist.

\section{Statistics}

For all statistical analyses, confirm that the following items are present in the figure legend, table legend, main text, or Methods section.

n/a Confirmed

$\square \bigotimes$ The exact sample size $(n)$ for each experimental group/condition, given as a discrete number and unit of measurement

$\square$ \A statement on whether measurements were taken from distinct samples or whether the same sample was measured repeatedly

$\square$ The statistical test(s) used AND whether they are one- or two-sided

$\square$ Only common tests should be described solely by name; describe more complex techniques in the Methods section.

$\bigotimes \square$ A description of all covariates tested

$\bigotimes \square$ A description of any assumptions or corrections, such as tests of normality and adjustment for multiple comparisons

$\square$ A full description of the statistical parameters including central tendency (e.g. means) or other basic estimates (e.g. regression coefficient)

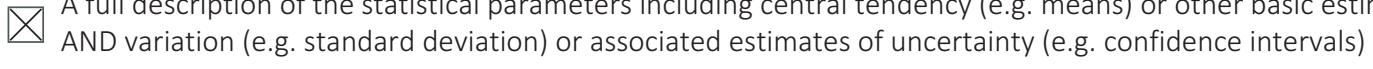

$\varnothing$ For null hypothesis testing, the test statistic (e.g. $F, t, r$ ) with confidence intervals, effect sizes, degrees of freedom and $P$ value noted

Give P values as exact values whenever suitable.

Х $\square$ For Bayesian analysis, information on the choice of priors and Markov chain Monte Carlo settings

$\bigotimes \square$ For hierarchical and complex designs, identification of the appropriate level for tests and full reporting of outcomes

Х Estimates of effect sizes (e.g. Cohen's $d$, Pearson's $r$ ), indicating how they were calculated

Our web collection on statistics for biologists contains articles on many of the points above.

\section{Software and code}

Policy information about availability of computer code

Data collection Flow cytometric analyses were performed with a BD FACSVerse, Violet(2), Blue(4), Red(2)

Quantitative PCR was performed with Applied Biosystems QuantStudio' 3 Real-Time PCR System

Pseudovirus luciferase activity was measured with BioTek Synergy H1 Hybrid Multi-Mode Microplate Reader

Data analysis $\quad$ Flow cytometric data were analysed with FlowJo v10.7.1

All other data were analysed and graphed using GraphPad Prism 8

For manuscripts utilizing custom algorithms or software that are central to the research but not yet described in published literature, software must be made available to editors/reviewers. We strongly encourage code deposition in a community repository (e.g. GitHub). See the Nature Research guidelines for submitting code \& software for further information.

\section{Data}

Policy information about availability of data

All manuscripts must include a data availability statement. This statement should provide the following information, where applicable:

- Accession codes, unique identifiers, or web links for publicly available datasets

- A list of figures that have associated raw data

- A description of any restrictions on data availability

All data related to Fig. 1a, b, Fig. 2b, c, e, f, h, i, Fig. 3, Extended Fig. 1a, d, Extended Fig. 3a, c-f, Extended Fig. 5, Extended Fig. 6 b are all included in Resource data and publicly available. 


\section{Field-specific reporting}

Please select the one below that is the best fit for your research. If you are not sure, read the appropriate sections before making your selection. $\bigotimes$ Life sciences $\quad \square$ Behavioural \& social sciences $\quad \square$ Ecological, evolutionary \& environmental sciences For a reference copy of the document with all sections, see nature.com/documents/nr-reporting-summary-flat.pdf

\section{Life sciences study design}

All studies must disclose on these points even when the disclosure is negative.

Sample size No sample size calculation was performed in advance. Rather numbers of mice analyzed were based on previous studies and on the numbers required to obtain statistical significance (Ref 14; Channappanvar et al, PMID: 31355779).

Data exclusions No data were excluded.

Replication All experiments were repeated at least twice with the same results. However, because of a shortage of mice, some measurements of immune parameters were performed once, with four mice/group.

Randomization Mice used in this study are inbred. Mice of the same gender and age were distributed randomly into different groups.

Blinding All observational data (Fig. 2 and extended Fig. 5) and histology analyses (Fig. 1c-j, Extended Fig. 1c, d, Extended Fig. 2, Extended Fig. 4 and Extended Fi. 6a) were obtained by blinded personnel and analyzed in a blinded fashion by a pathologist, to avoid subjective bias.

\section{Reporting for specific materials, systems and methods}

We require information from authors about some types of materials, experimental systems and methods used in many studies. Here, indicate whether each material, system or method listed is relevant to your study. If you are not sure if a list item applies to your research, read the appropriate section before selecting a response.

Materials \& experimental systems

\begin{tabular}{l|l}
\hline$n / a$ & Involved in the study \\
$\square$ & $\bigotimes$ Antibodies \\
$\square$ & $\bigotimes$ Eukaryotic cell lines \\
$\square$ & $\square$ Palaeontology \\
$\square$ & $\square$ Animals and other organisms \\
$\square$ Clinical data
\end{tabular}

Methods $\mathrm{n} / \mathrm{a}$ Involved in the study

\ $\square$ ChIP-seq

$\square$ Flow cytometry

Х $\square$ MRI-based neuroimaging

\section{Antibodies}

Antibodies used

For flow cytometry: Anti-mouse CD3e-BV421 (clone 145-2C11, Cat. No.: 562600), Anti-mouse CD16/32 (clone 93, Cat. No.:101302), Anti-mouse CD4-PercP (clone RM4-5, Cat. No.: 550954), Anti-mouse CD8-APCCy7 (clone 53-6.7, Cat. No.: 100714), Anti-mouse CD220-APC (clone RA3-6B2, Cat. No.: 553092), Anti-mouse Ly6C-PerCP (clone HK1.4, Cat. No.: 128028), anti-mouse Ly6G-FITC (clone 1A8, Cat. No.: 127606), Antimouse CD11b-BV510 (clone M1/70, Cat. No.: 101263), Anti-mouse CD11c-BV421 (clone N418, Cat. No.:

117343), Anti-mouse CD64-PE-Cy7 (X54-5/7.1, Cat. No.: 139314), all from Biolegend, San Diego, CA; Antimouse CD103-APC (clone 2E7, Cat. No.: 17-1031-80), Anti-mouse TNF-FITC (clone MP6-XT22, Cat. No.:

11-7321-82), Anti-mouse IFN- - -APC (clone XMG1.2, Cat. No.: 25-7311-82), all from eBioscience, San Diego,

CA.

For IHC: Rabbit monoclonal antibody recognizing SARS-CoV-2 N protein (Cat. No.:40143-R019), Sino Biological US Inc.

Validation

All antibodies were obtained commercially. Specificity and sensitivity were validated by the manufacturers.

\section{Eukaryotic cell lines}

Policy information about cell lines

Cell line source(s)

Calu-3 (ATCC HTB-55) and Vero E6 cells (ATCC CRL-1586)

Authentication

None of the cells were formally authenticated although they remained sensitive to infection with SARS-CoV-2. 
Mycoplasma contamination

Commonly misidentified lines (See ICLAC register)

\section{Negative for Mycoplasma}

No commonly misidentified cell lines were used.

\section{Animals and other organisms}

Policy information about studies involving animals; ARRIVE guidelines recommended for reporting animal research

Laboratory animals

Wild animals

Field-collected samples

Ethics oversight
7-8 weeks old Male and female C57/BL6 K1 8-hACE2 mice

none

none

Note that full information on the approval of the study protocol must also be provided in the manuscript.

\section{Human research participants}

Policy information about studies involving human research participants

Population characteristics

The convalescent plasma donor was a 58 year old female who had molecularly confirmed COVID-19 more than 4 weeks prior to their donation. Following the donation, she tested positive for HLA antibodies so the plasma was not eligible for administration to patients and was diverted to research. Antibody testing (EUROIMMUN SARS-COV-2 ELISA (IgG)) performed on this donor was 9.8, well above the cutoff of 1.1 for a positive result. Neutralization titer using a luciferase-expressing SARS-CoV-2 S protein pseudovirus assay showed that the neutralization IC50 titer was 1:1,480. Control plasma was obtained from an expired plasma unit collected prior to COVID-19 spread in our area.

Recruitment

Written informed consent was obtained from subjects to obtain plasma for participation in this study.

Ethics oversight

High titer convalescent and control plasma was collected with subject consent under IRB (\#202003554) and (\#201402735) approved protocols respectively that allow use of samples for research.

Note that full information on the approval of the study protocol must also be provided in the manuscript.

\section{Flow Cytometry}

Plots

Confirm that:

$\bigotimes$ The axis labels state the marker and fluorochrome used (e.g. CD4-FITC).

$\bigotimes$ The axis scales are clearly visible. Include numbers along axes only for bottom left plot of group (a 'group' is an analysis of identical markers).

$\triangle$ All plots are contour plots with outliers or pseudocolor plots.

$\triangle$ A numerical value for number of cells or percentage (with statistics) is provided.

\section{Methodology}

Sample preparation

Instrument

Software

Cell population abundance
Mice were anesthetized with ketamine/xylazine before dissection for lung harvest. Lungs were perfused transcardially and minced into smaller pieces with a pair of surgical scissors. The minced lungs were left in digestion buffer $(1 \mathrm{mg} / \mathrm{ml}$ collagenase D (Roche) and $0.1 \mathrm{mg} / \mathrm{ml}$ DNase I (Roche)) at $37^{\circ} \mathrm{C}$ for 30 minutes. Digested tissue was passed through a $70 \mu \mathrm{m}$ cell strainer and cells were pelleted by centrifugation at 330 RCF for 5 minutes. Cell pellet was resuspended in either FACS buffer or RPMI for peptide stimulation. Fc receptors on cells were blocked and cells were subsequently fixed with Cytofix/ cytoperm followed by intracellular IFN $Y$ and TNF staining after peptide stimulation. Cells were pelleted and resuspended in FACS buffer for analysis.

BD FACSVerse, Violet(2), Blue(4), Red(2)

FlowJo v10.7.1

Total CD4 T cell frequency in lungs ranged from $3.87 \%$ to $8.57 \%$ of live single cells (Virus-specific CD4+ T cell frequency in lungs ranged from $0 \%$ to $11.15 \%$ of $\mathrm{CD} 4 \mathrm{~T}$ cells). $0 \%$ frequency was found in uninfected mice.

Total CD8 T cell frequency in lungs ranged from $1.96 \%$ to $11.83 \%$ of live single cells (Virus-specific CD8+ T cell frequency in lungs ranged from $0 \%$ to $9.37 \%$ in CD8 T cells). $0 \%$ frequency was found in uninfected mice.

B cell frequency in lungs ranged from $4.33 \%$ to $11.52 \%$ of live single cells

Total DC frequency in lungs ranged from $2.10 \%$ to $18.32 \%$ of live single cells

CD103+ DC frequency in lungs ranged from $0.04 \%$ to $14.53 \%$ of live single cells

PMN frequency from lungs ranged from $0.17 \%$ to $4.58 \%$ of live single cells 
Inflammatory monocyte/macrophage frequency in lungs ranged from $0.75 \%$ to $18.90 \%$ of live single cells Alveolar macrophage frequency in lungs ranged from $7.65 \%$ to $13.5 \%$ of live single cells

Gating strategy

Lymphocytes were gated using SSC-A vs FSC-A .Single cells were gated using FSC-A vs FSC-H. B and T cells were gated using B220 and CD3, respectively, as B220+ and CD3+ populations from the single cell population. CD8+ and CD4+ T cells were gated after selection of CD3+ T cell populations. AM and DC were gated on CD11c vs CD64 from the single cell gate as CD11c + CD64+ and CD11C+ CD64- populations respectively. CD103+ DC were gated on the DC population using FCS-A vs CD103. Neutrophils were gated using Ly6G vs CD11c on the single cell gate and were identified as Ly6G+ CD11c intermediate populations. IMMs were gated on the single cell population using Ly6C vs CD11b (Ly6C+CD11b+ population).

$\bigotimes$ Tick this box to confirm that a figure exemplifying the gating strategy is provided in the Supplementary Information. 